First passages for a search by a swarm of independent random searchers

This article has been downloaded from IOPscience. Please scroll down to see the full text article.

J. Stat. Mech. (2011) P06022

(http://iopscience.iop.org/1742-5468/2011/06/P06022)

View the table of contents for this issue, or go to the journal homepage for more

Download details:

IP Address: 138.100.137.103

The article was downloaded on 04/07/2011 at 10:36

Please note that terms and conditions apply. 


\title{
First passages for a search by a swarm of independent random searchers
}

\author{
Carlos Mejía-Monasterio ${ }^{1}$, Gleb Oshanin ${ }^{2}$ \\ and Grégory Schehr ${ }^{3}$
}

${ }^{1}$ Laboratory of Physical Properties, Technical University of Madrid, Av. Complutense s/n, E-28040 Madrid, Spain

${ }^{2}$ Laboratoire de Physique Théorique de la Matière Condensée (UMR CNRS 7600), Université Pierre et Marie Curie/CNRS, 4 place Jussieu, F-75252 Paris Cedex 5, France

${ }^{3}$ Laboratoire de Physique Théorique (UMR CNRS 8627), Bâtiment 210, Université Paris-Sud 11, 91405 Orsay Cedex, France

E-mail: carlos.mejia@upm.es, oshanin@lptmc.jussieu.fr and gregory.schehr@th.u-psud.fr

Received 19 May 2011

Accepted 10 June 2011

Published 30 June 2011

Online at stacks.iop.org/JSTAT/2011/P06022

doi:10.1088/1742-5468/2011/06/P06022

\begin{abstract}
In this paper we study some aspects of search for an immobile target by a swarm of $N$ non-communicating, randomly moving searchers (numbered by the index $k, k=1,2, \ldots, N$ ), which all start their random motion simultaneously at the same point in space. For each realization of the search process, we record the unordered set of time moments $\left\{\tau_{k}\right\}$, where $\tau_{k}$ is the time of the first passage of the $k$ th searcher to the location of the target. Clearly, $\tau_{k} \mathrm{~s}$ are independent, identically distributed random variables with the same distribution function $\Psi(\tau)$. We evaluate then the distribution $P(\omega)$ of the random variable $\omega \sim \tau_{1} / \bar{\tau}$, where $\bar{\tau}=N^{-1} \sum_{k=1}^{N} \tau_{k}$ is the ensemble-averaged realization-dependent first passage time. We show that $P(\omega)$ exhibits quite a non-trivial and sometimes a counterintuitive behavior. We demonstrate that in some well-studied cases (e.g. Brownian motion in finite $d$-dimensional domains) the mean first passage time is not a robust measure of the search efficiency, despite the fact that $\Psi(\tau)$ has moments of arbitrary order. This implies, in particular, that even in this simplest case (not to mention complex systems and/or anomalous diffusion) first passage data extracted from a single-particle tracking should be regarded with appropriate caution because of the significant sample-to-sample fluctuations.
\end{abstract}


Keywords: fluctuations (theory), stochastic search, Brownian motion, extreme value statistics

ArXiv ePrint: $1106.4182 \mathrm{v} 1$

\section{Contents}

Introduction

1. First passage to the target for $N$ independent searchers $\quad 7$

2. General form of $P(\omega)$ for heavy-tailed first passage time distributions 8

3. Exactly solvable cases for the heavy-tailed distributions 10

3.1. Two non-communicating random searchers . . . . . . . . . . . . . . . 10

3.2. Three non-communicating random searchers . . . . . . . . . . . . . 11

3.3. $N$ non-communicating random searchers for $\mu=1 / 2$ and $3 / 2 \ldots \ldots$. . . 12

4. Asymptotic large- $N$ behavior of $P(\omega)$ for heavy-tailed first passage time distributions

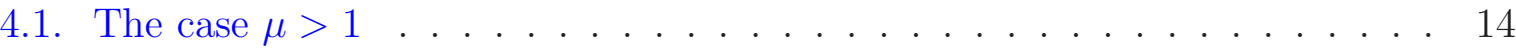

4.2. The case $\mu=1 \ldots \ldots \ldots \ldots \ldots \ldots$. . . . . . . . . . . . . . . . . . . . . 14

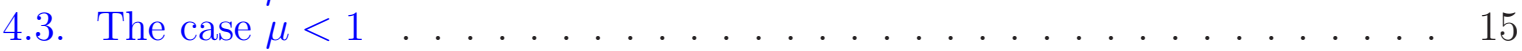

5. The distribution $P(\omega)$ for exponentially truncated first passage time distributions. Two non-communicating searchers 16

5.1. Two independent BMs in a semi-infinite $1 \mathrm{D}$ system with a bias . . . . . . . 17

5.2. Two independent unbiased BMs on a finite interval . . . . . . . . . . . . 18

5.3. Two independent BMs in a disc with a reflecting boundary . . . . . . . . . 22

5.4. Two independent BMs in a sphere with a reflecting boundary . . . . . . . 24

5.5. Two independent BMs in a sphere with an adsorbing boundary . . . . . . 26

6. The distribution $P(\omega)$ for exponentially truncated first passage time distributions. Three and more non-communicating searchers 28

7. Conclusions 30

Acknowledgments $\quad 33$

References $\quad 33$

\section{Introduction}

Search processes are ubiquitous in Nature: in order to survive, predators have to hunt the prey and the prey have to forage [1]-[3]. In order to convert into required reaction products, the reactants involved in chemical or biochemical reactions have first to find each other [4]. In many biophysical processes ligands search for binding sites [5,6], proteins seek the target sequences on DNA's [7], etc. Human beings look for a better job, partners, shelter, files in databases, etc. Even an attempt to unlock a pin-protected device can be considered as a search in the space of all possible passwords [8].

Search for a desired target depends generally on a variety of different conditions and may take place in different environments: targets may be sparse, hidden or difficult to detect even when found. The targets may be immobile or mobile, try to avoid searchers 
or to evade from the searched area $[9,10]$. They may have no or they may have their own lifetime and vanish before they are detected.

Searchers may be immobile, as happens, e.g., in a visual search [11], may move freely or may interact with the environment. Their motion may be hindered under conditions of molecular crowding [12], e.g. in a cell's cytoplasm or in dynamical backgrounds formed by other randomly moving particles, or facilitated due to interactions with molecular motors. The searchers may search 'blindly', detecting the target only upon an encounter with it, or 'smell' (or 'see') the target somehow at long distances correcting their motion $[9,13]$. They may have no memory of previously visited areas or adapt their strategy 'on-line', repelling themselves from their footprints on the searched substrate. Finally, the searchers may act individually or in swarms [14]-[16].

In general, for each specific situation different search strategies may be realized, and the question of efficient ones has motivated a great deal of work in the last few years. While earlier works have considered deterministic search algorithms (see, e.g., $[1,2,17]$ and references therein) specific to such human activities as, say, a search for natural resources or rescue operations, more recent studies focused on random search strategies. It was realized that the strategies based on Lévy flights or walks $[3,18,19]$, in which a searcher performs excursions whose lengths are random variables with heavy-tailed distributions, in some aspects are more advantageous than a search based on conventional Brownian motion, or on random walks which step on nearest neighbors only. Naturally, in this case the large-scale dynamics of searchers is superdiffusive.

Following the observation of trajectories of foraging animals in which active local search phases randomly alternate with relocation phases (see, e.g., [20]) another type of random search - an intermittent search - has been proposed. In this algorithm the search process is characterized by two distinct types of motion-a ballistic relocation stage when the searcher is non-receptive to the target and a relatively slow phase with a random Brownian-type motion when the target may be detected [21]-[24]. Much effort has been invested recently in understanding different optimization schemes for such a random search. In particular, one looked for the conditions allowing us to minimize the mean first passage time for the process which is unlimited in time [21], or sought to enhance the chances of successful detection by minimizing the non-detection probability for the search process constrained to happen within a finite time interval [22]-[24]. Note that for such a search the large-scale dynamics is diffusive, albeit intermittent.

Finally, a combination of a Lévy-based and intermittent search has been proposed in [25], in which the length of the relocation stage was taken as a random variable with a heavy-tailed distribution. It was shown that such a combined strategy is advantageous in the critical case of rare targets.

In this paper we discuss some aspects of a blind search by a swarm of $N$ independent, non-communicating searchers. We consider a situation, as depicted in figure 1 , in which $N$ searchers (numbered by the index $k, k=1,2, \ldots, N$ ) occupy initially the same position in space, at some distance $x_{0}$ apart from an immobile target, start their random motion simultaneously and arrive for the first time to the location of the target at times $\tau_{k}$, respectively. Note that $\tau_{k} \mathrm{~s}$ are not ordered. Clearly, in such a situation the first passage times $\tau_{k}$ s are independent, identically distributed random variables with the same distribution $\Psi(\tau)$. 
First passages for a search by a swarm of independent random searchers

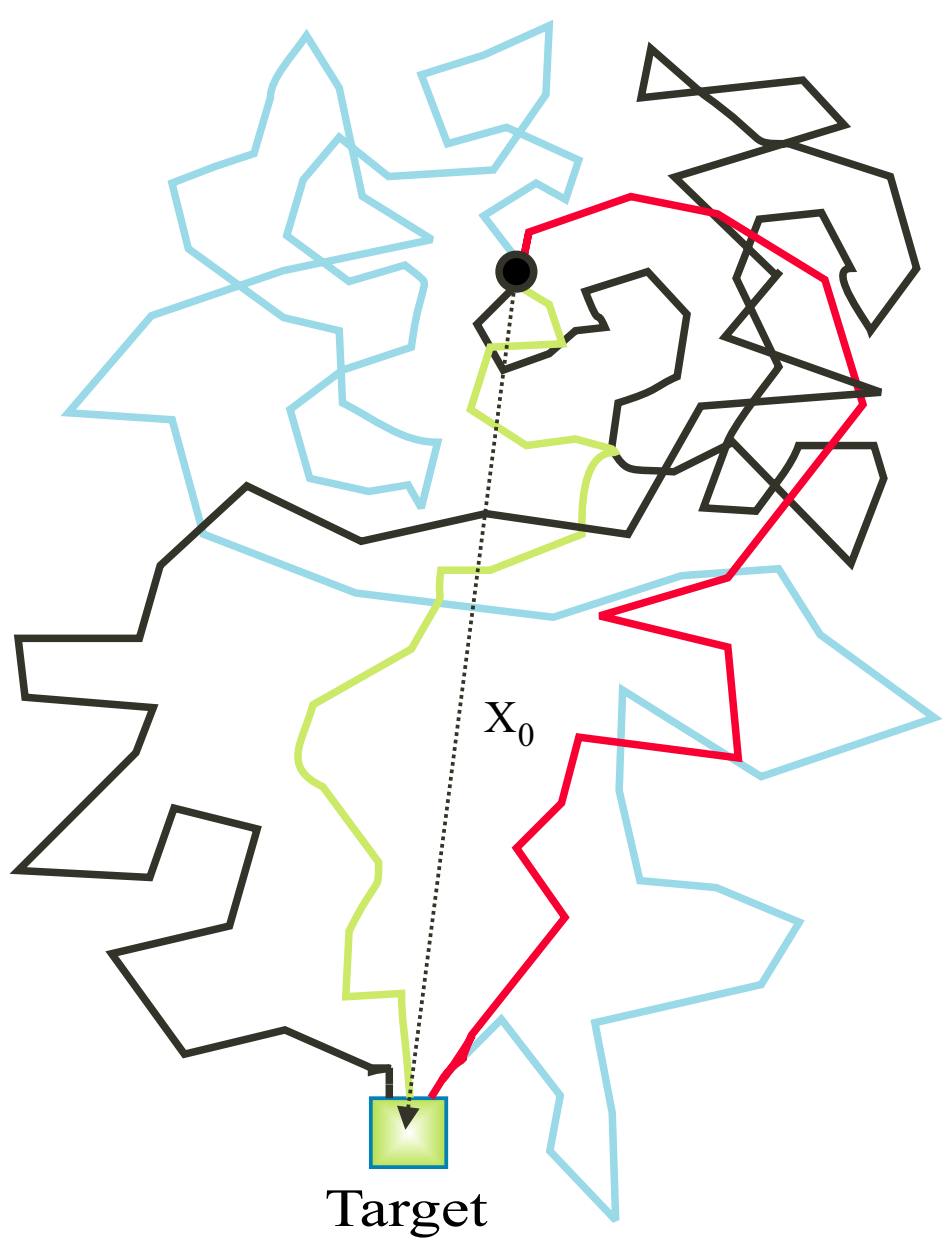

Figure 1. A sketch of trajectories of four searchers starting at the same point (filled black circle) at a distance $x_{0}$ from the target and reaching the target for the first time at different time moments.

We focus here on the random variable

$$
\omega=\frac{1}{N} \frac{\tau_{1}}{\bar{\tau}}
$$

where $\bar{\tau}$ is the averaged, over the ensemble of $N$ searchers, realization-dependent first passage time:

$$
\bar{\tau}=\frac{1}{N} \sum_{k=1}^{N} \tau_{k} .
$$

Hence, the random variable $\omega$ probes the first passage time of a given searcher relative to the ensemble-averaged first passage time for $N$ independent searchers. The scaling factor $1 / N$ in equation (1) is introduced here for convenience, so that regardless of the value of $N$, the random variable $\omega$ has a support on $[0,1]$. We note parenthetically that random variables such as in equation (1) were previously studied in [26]-[31] within a different context. 
Our goal is to calculate the distribution function

$$
P(\omega)=\left\langle\delta\left(\omega-\frac{1}{N} \frac{\tau_{1}}{\bar{\tau}}\right)\right\rangle,
$$

where $\delta(\cdot)$ is the delta function and the angular brackets denote averaging over different realizations of independent, identically distributed random variables $\tau_{k}$.

For arbitrary normalized $\Psi(\tau)$, the distribution $P(\omega)$ is normalized, possesses all moments and the first moment $\langle\omega\rangle \equiv \int_{0}^{1} \omega \mathrm{d} \omega P(\omega)=1 / N$. Note that $P(\omega)$ can be seen as a measure of the robustness of a given search algorithm. Clearly, if $P(\omega)$ appears to be sharply peaked at $\omega=1 / N$, this would signify that the underlying search algorithm is quite robust. Otherwise, if the distribution appears to be broad, or even to have a multi-modal shape, this would imply that the performance of such an algorithm is rather poor and sample-to-sample fluctuations matter. From yet another conceptual perspective, one can say that $P(\omega)$ probes the validity of the mean first passage time as the proper measure of a search process efficiency. We proceed to show that in many situations $P(\omega)$ has a rather complicated structure of which the mean behavior is not representative. We will show that in different situations $P(\omega)$ may have completely different shapes (modality) and also change the shape (say, from a unimodal bell-shaped form to a bimodal U- or an M-shaped one) when some of the parameters are slightly modified. A similar phenomenon of a shape reversal has been previously observed for different mathematical objects in $[27,30,31]$.

We turn next to the key feature: the distribution $\Psi(\tau)$ of $\tau_{k}$ s, which encodes all the information on the specific properties of the searchers' random motion and on their initial location relative to the target. Without a significant lack of generality, we suppose that for a search in infinitely large systems, in which all searchers perform a blind search and detect the target with probability 1 upon a first encounter with it, $\Psi(\tau)$ can be defined as

$$
\Psi(\tau)=\frac{a^{\mu}}{\Gamma(\mu)} \exp \left(-\frac{a}{\tau}\right) \frac{1}{\tau^{1+\mu}},
$$

where $\Gamma(\cdot)$ is the gamma function, $a$ is a characteristic parameter which sets the cutoff of the distribution at small values of $\tau$ and $\mu \geq 0$ is the so-called persistence exponent [32]. Note that, depending on the dimensionality of space and type of random motion, one can encounter completely different values of $\mu ; \mu$ can be $0 \leq \mu<1, \mu=1$ or $\mu>1$. The distribution in equation (4) is normalized but does not possess already a first moment (i.e. the mean first passage time) for $0 \leq \mu<1$.

The distribution in equation (4) is exact for a Brownian motion (BM) in semi-infinite one-dimensional (1D) systems, in which case $\mu=1 / 2$ and $a=x_{0}^{2} / 4 D, D$ being the diffusion coefficient [33]. In fact, the case $\mu=1 / 2$ appears to be very representative. According to the theorem due to Sparre Andersen [34], in 1D systems for any discrete-time random walk with each step length chosen from a continuous, symmetric but otherwise arbitrary distribution the first passage time distribution $\Psi(n)$ decays with a number of steps $n$ as $n^{-3 / 2}$. For continuous-time $\tau$ Markov processes, an analogous result is $\Psi(\tau) \sim \tau^{-3 / 2}$. This universality, of course, is broken when subordination effects (i.e. long-tailed waiting time distributions in a CTRW sense) are present.

Although not exact, the distribution in equation (4) is physically quite plausible for other types of random motion in infinite 1D systems provided that an appropriate choice 
of the exponent $\mu$ is made [33]: in particular, equation (4) with $\mu=1-1 / \alpha$ is a reasonable approximation for the first-arrival probability density for Lévy flights with Lévy index $\alpha$, $1<\alpha<2$ [35] and with $\mu=1-H[36]$-for the first passage time distribution for fractional BM with Hurst index $H, 0<H<1$. One may also claim that $\mu=1-d_{\mathrm{f}} / d_{\mathrm{w}}$ for $d_{\mathrm{f}}<d_{\mathrm{w}}$ (compact exploration [37]), where $d_{\mathrm{f}}$ is a non-integer spatial dimension and $d_{\mathrm{w}}$ is the fractal dimension of random motion trajectories, since the first passage distribution is defined as the time derivative of the survival probability of an immobile target or of the normalized current through the surface of the target [33]. Hence, its long-time tail is the same as the long-time tail of the time derivative of the Smoluchowski constant [38, 39].

The case $\mu=0$ can be encountered in borderline situations of compact exploration, when the dimension of space $d_{\mathrm{f}}$ equals the fractal dimension $d_{\mathrm{w}}$ of the random motion trajectories. In particular, this situation is realized for standard BM $\left(d_{\mathrm{w}}=2\right)$ in twodimensional (2D) space. Here, one finds that the long-time tail of the distribution $\Psi(\tau)$ follows (see, e.g., [33])

$$
\Psi(\tau) \sim \frac{1}{\tau \ln ^{2}(\tau)}
$$

This situation will be considered in more detail in what follows.

Finally, one finds $\mu=d / 2-1$ (or, more generally, $\mu=d_{\mathrm{f}} / d_{\mathrm{w}}-1$ ) for Brownian (anomalous) motion in $d>2\left(d_{\mathrm{f}}>d_{\mathrm{w}}\right)$-dimensional systems. Note, however, that here the expression in equation (4) is not normalized and thus cannot be considered as a distribution since only a finite fraction of trajectories will visit the target within an infinite time so that the searchers will have a finite probability to escape to infinity (non-compact exploration [37]). One can, however, normalize $\Psi(\tau)$ in equation (4) by hand, treating it as the conditional probability distribution of the first passage times for such trajectories which visit the target within an infinite time.

Next, we will consider a normalized, exponentially truncated version of the distribution in equation (4):

$$
\Psi(\tau)=\frac{(a b)^{\mu / 2}}{2 K_{\mu}(2 \sqrt{a / b})} \exp \left(-\frac{a}{\tau}\right) \frac{1}{\tau^{1+\mu}} \exp \left(-\frac{\tau}{b}\right),
$$

where $K_{\mu}(\cdot)$ is the modified Bessel function. In contrast to $\Psi(\tau)$ in equation (4), the distribution in equation (6) possesses moments of arbitrary order. Note that the latter point is crucial and, according to a common belief, the first moment of the distribution, i.e. the mean first passage time, can be regarded as a robust measure of the search process efficiency. We set out to show that in many situations this is not the case due to the significant sample-to-sample fluctuations.

The distribution in equation (6) is exact for a BM in semi-infinite $1 \mathrm{D}$ systems in the presence of a constant bias pointing toward the target (see, e.g., [33]). In this case one has $a=x_{0}^{2} / 4 D$, where $x_{0}$ is the starting point and $b=4 D / v^{2}, v$ being the drift velocity.

One may argue, as well, that the expression in equation (6) is an appropriate approximation for the first passage time distribution for search in finite systems or search assisted by smell. In general, of course, for random motion in finite systems the distribution $\Psi(\tau)$ will be represented as a series of exponentials; the form in equation (6) is thus tantamount to a heuristic approximation of this series in which one takes a behavior specific to an infinite system and truncates it by an exponential function with the 
characteristic decay time equal to the largest relaxation time. Depending on a particular situation, the cutoff parameter $b$ will be either proportional to the volume of the system (for BM in $d>2$ systems), to $L^{2}$ where $L$ is the length of the interval for BM in 1D, to $S \ln (S)$ for BM in finite 2D systems of area $S$, or inversely proportional to the strength of the bias for biased diffusion. Some of these situations will be discussed in detail in the following.

We finally remark that the distribution in equation (6) appears in many other physical problems. To name but a few we mention the distribution of times between action potentials (or the ISI distribution) in the integrate-and-fire model of neuron dynamics [40], the distribution of the stopping distances for the sliding motion of a solid block on an inclined heterogeneous plane [41], the avalanche lifetime distribution in the mean-field version of the Bak-Sneppen model [42], the distribution of the probability current in finite disordered one-dimensional samples [43] or the distribution of the number of times that a particle diffusing in a sphere hits its boundary during some time interval [6]. Thus our subsequent analysis applies to these systems as well.

The outline of our paper is as follows: we start in section 1 with a general remark on the tails of the first passage time distribution in the case with $N$ independent randomly moving searchers. In section 2 we derive an exact result for the distribution $P(\omega)$ for $\Psi(\tau)$ in equation (4) with arbitrary $\mu$ and for arbitrary $N$. In section 3 we discuss several exactly solvable particular cases. Section 4 is devoted to the asymptotic analysis of $P(\omega)$ for large $N$ and arbitrary $\mu$. In section 5 we evaluate $P(\omega)$ for the exponentially truncated distribution in equation (6) in the case of two searchers. We also furnish here exact calculations of $P(\omega)$ for two BMs in finite 1D, 2D and 3D spherical domains. Next, in section 6 , we discuss the form of $P(\omega)$ for $N$ searchers whose first passage time distribution is given by equation (6). Finally, in section 7 we conclude with a summary of our results and some generalizations.

\section{First passage to the target for $N$ independent searchers}

We begin with a somewhat evident but conceptually very important remark on the tails of the first passage time distribution in the case of $N$ independent, non-communicating randomly moving searchers. For the original results and a discussion we address the reader to [44]. Some other interesting aspects of this model were discussed in [15, 45].

Let $P_{N}(\tau)$ denote the probability that up to time moment $\tau$ neither of $N$ searchers has visited the target, i.e. the target remained non-detected up to time $\tau$. For the situation under consideration, clearly

$$
P_{N}(\tau)=P_{1}(\tau)^{N}
$$

where $P_{1}(\tau)$ is an analogous probability for a single searcher. Supposing that the target is found as soon as any of the searchers arrives to its location for the first time, we have that for $N$ searchers the first passage density $\Psi_{N}(\tau)$ is given by

$$
\Psi_{N}(\tau)=-\frac{\mathrm{d} P_{N}(\tau)}{\mathrm{d} \tau}
$$

This yields immediately that $\Psi_{N}(\tau)$ follows (for the parental distribution in equation (4)), as $\tau \rightarrow \infty$ :

$$
\Psi_{N}(\tau) \sim \frac{1}{\tau^{1+\mu N}}
$$


A remarkable feature of this simple result is that, depending on the number of searchers $N$, the distribution in equation (9) may have finite moments even if $\mu \leq 1$, contrary to the parent distribution in equation (4) which does not have any moment for such values of $\mu$. For instance, for $0<\mu<1$ and $\mu N>1$, equation (9) has a finite first moment, i.e. a finite mean first passage time, for $\mu N>2$ it has a finite second moment, and generally, for $\mu N>k$ it has $k$ first finite moments. This is a crucially important advantage of search processes involving $N$ searchers, which makes the search process more efficient even for non-communicating searchers.

Next, it might be instructive to consider some exactly solvable case, i.e. BM in semiinfinite $1 \mathrm{D}$ systems, and to calculate the $N$ dependence of the mean first passage time. In this case, the probability that a single searcher has not visited the location of the target (the origin) up to time moment $\tau$, starting at distance $x_{0}$ from the target, obeys $P_{1}(\tau)=\operatorname{erf}\left(x_{0} / \sqrt{4 D \tau}\right)$, where $\operatorname{erf}(\cdot)$ is the error function. Hence, the first moment of the distribution in equation (8), i.e. the mean first passage time $\left\langle\tau_{N}\right\rangle$ for an ensemble of $N$ independent searchers is defined as

$$
\left\langle\tau_{N}\right\rangle=-\int_{0}^{\infty} \mathrm{d} \tau \tau \frac{\mathrm{d} P_{N}(\tau)}{\mathrm{d} \tau}=\int_{0}^{\infty} \mathrm{d} \tau \operatorname{erf}^{N}\left(\frac{x_{0}}{\sqrt{4 D \tau}}\right) .
$$

The integral in the latter equation is convergent, as we have already remarked, for $N \geq 3$. One verifies that $\left\langle t_{N}\right\rangle$ is a slowly decreasing function of the number of searchers $N$, and, for $N \gg 1$, we find that

$$
\left\langle\tau_{N}\right\rangle \sim \frac{x_{0}^{2}}{4 D \ln (N)}
$$

Therefore, the more searchers one has, the less the mean first passage time is. Curiously enough, this essentially 1D result, $\left\langle\tau_{N}\right\rangle$ in equation (11), coincides exactly with the mean residence time which $N$ BMs spend simultaneously together in a circular disc of radius $x_{0}$ on a $2 \mathrm{D}$ plane within an infinite time interval [46], or with the mean first exit time of one of $N$ BMs from such a disc [47].

Note finally that the mean first passage time may acquire a much stronger dependence on $N$ and decrease much faster for communicating searchers which share the information on the location of the target [14]. Note, as well, that increasing the number of 'searchers' allows us to reduce substantially the time necessary to reach the target site on a DNA for proteins which strongly bind to other nonspecific sites acting as deep temporal traps [48]. Some properties of the first passage time distribution for the event in which $N$ random walks appear for the first time simultaneously at the same lattice site have been discussed in [9] within the context of a survival of an evasive prey.

\section{General form of $P(\omega)$ for heavy-tailed first passage time distributions}

Let $\langle\exp (-\lambda \omega)\rangle, \lambda \geq 0$, denote the moment generating function of the random variable $\omega$, equation (1). For arbitrary $\Psi(\tau)$, it can be formally represented as an $N$-fold integral:

$\left\langle\mathrm{e}^{-\lambda \omega}\right\rangle=\int_{0}^{\infty} \cdots \int_{0}^{\infty}\left(\prod_{n=1}^{N} \mathrm{~d} \tau_{n} \Psi\left(\tau_{n}\right)\right) \exp \left(-\lambda \frac{\tau_{1}}{\tau_{1}+\tau_{2}+\cdots+\tau_{N}}\right)$. 
Integrating over $\mathrm{d} \tau_{1}$, we change the integration variable $\tau_{1} \rightarrow \omega$ to get

$$
\begin{aligned}
\left\langle\mathrm{e}^{-\lambda \omega}\right\rangle=\int_{0}^{1} & \frac{\mathrm{d} \omega}{(1-\omega)^{2}} \mathrm{e}^{-\lambda \omega} \int_{0}^{\infty} \cdots \int_{0}^{\infty}\left(\prod_{n=2}^{N} \mathrm{~d} \tau_{n} \Psi\left(\tau_{n}\right)\right) \\
& \times\left(\tau_{2}+\cdots+\tau_{N}\right) \Psi\left(\frac{\omega}{1-\omega}\left(\tau_{2}+\cdots+\tau_{N}\right)\right) .
\end{aligned}
$$

Using next the following integral representation:

$$
\tau \Psi(\tau)=\int_{0}^{\infty} \mathrm{d} p Q(p) \mathrm{e}^{-p \tau}
$$

where the kernel $Q(p)$ is some unknown function defined via the inverse Laplace transform of the distribution $\Psi(\tau)$, we obtain the following general result for the probability density $P(\omega)$ in the case of $N$ identical $\tau$ variables:

$$
P(\omega)=\frac{1}{\omega^{2}} \int_{0}^{\infty} \mathrm{d} \lambda Q\left(\frac{1-\omega}{\omega} \lambda\right) \Phi^{N-1}(\lambda),
$$

with $\Phi(\lambda)=\langle\exp (-\lambda \tau)\rangle$ being the characteristic function of the distribution $\Psi(\tau)$.

Now, for the distribution in equation (4) we have

$$
Q(p)=\frac{a^{(\mu+1) / 2}}{\Gamma(\mu)} p^{(\mu-1) / 2} J_{\mu-1}(2 \sqrt{a p})
$$

and

$$
\Phi(\lambda)=\frac{2 a^{\mu / 2}}{\Gamma(\mu)} \lambda^{\mu / 2} K_{\mu}(2 \sqrt{a \lambda})
$$

where $J_{\nu}(\cdot)$ is the Bessel function.

Substituting the expressions in equations (16) and (17) into equation (15), we find that the probability density $P(\omega)$ is given by

$$
P(\omega)=\frac{2^{-\mu}}{\Gamma(\mu)} \frac{(1-\omega)^{(\mu-1) / 2}}{\omega^{(3+\mu) / 2}} \int_{0}^{\infty} \mathrm{d} u u^{\mu} J_{\mu-1}\left(\sqrt{\frac{1-\omega}{\omega}} u\right) \Xi^{N-1}(u),
$$

independently of $a$, with

$$
\Xi(u)=\frac{2^{1-\mu}}{\Gamma(\mu)} u^{\mu} K_{\mu}(u) .
$$

The result in equation (18) defines an exact distribution $P(\omega)$ for arbitrary $\mu$ and $N$. In several particular cases, the integral in equation (18) can be performed in closed form: when $N=2$ or 3 and arbitrary $\mu>0$, or when $\mu$ is equal to half of an odd integer, while $N$ is arbitrary. We discuss below some of these cases, as well as present an asymptotic analysis of $P(\omega)$ in the limit $N \gg 1$. 


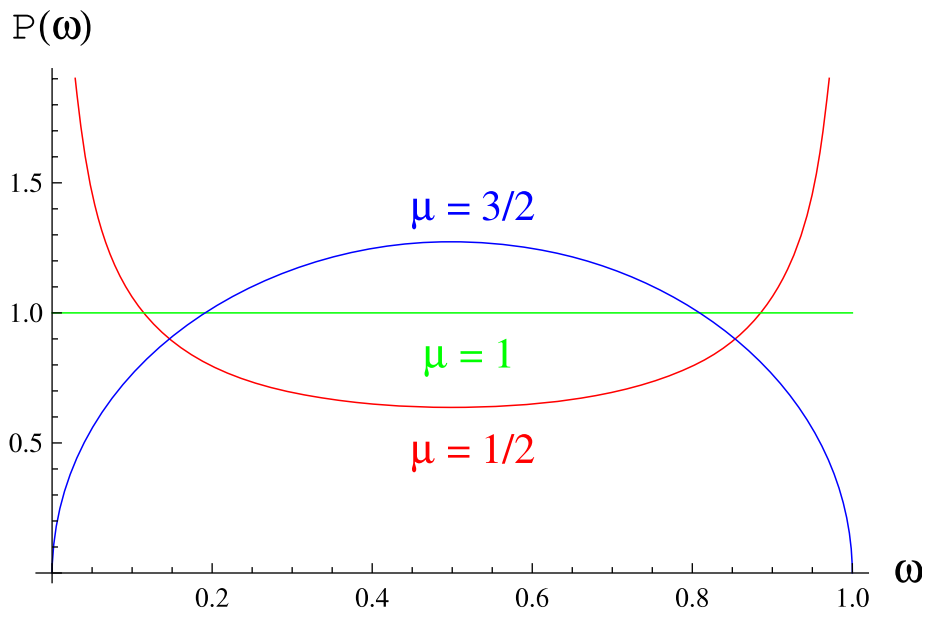

Figure 2. The distribution $P(\omega)$ in equation (20) for $\mu=1 / 2,1$ and $3 / 2$.

\section{Exactly solvable cases for the heavy-tailed distributions}

\subsection{Two non-communicating random searchers}

Consider first the case of just two random searchers, $N=2$. For this simple situation, one readily finds from equation (18) that $P(\omega)$ is given explicitly by

$$
P(\omega)=\frac{\Gamma(2 \mu)}{\Gamma^{2}(\mu)} \omega^{\mu-1}(1-\omega)^{\mu-1},
$$

i.e. in this case $P(\omega)$ is a beta distribution. This result has been also obtained within a different context in [26].

Notice now that, despite its simplicity, the result in equation (20) contains a surprise: it has a completely different shape (modality) depending on whether $0<\mu<1, \mu=1$ or $\mu>1$ (see figure 2). When $0<\mu<1$, the distribution $P(\omega)$ has a characteristic $U$ shape, so that the most probable values of $\omega$ are 0 and 1 . Strikingly, the mean $\langle\omega\rangle=1 / 2$ corresponds in this case to the least probable value of the distribution. This signifies that in this case there is no symmetry between two identical searchers and both arrive to the target for the first time at distinctly different times. Note that for $\mu=1 / 2$ (two BMs in $1 \mathrm{D}), P(\omega)=1 / \pi \sqrt{\omega(1-\omega)}$ and hence the probability $P(\omega \leq \Omega)$ that $\omega$ attains any value from the interval $[0, \Omega]$ obeys

$$
P(\omega \leq \Omega)=\frac{2}{\pi} \arcsin (\sqrt{\Omega})
$$

which is the continuous arcsine distribution.

When $\mu=1$, the distribution $P(\omega)$ in equation (20) is uniform, which means that for either of the searchers its first passage time, relative to the average over the ensemble of two searchers, may take any value with equal probability.

Finally, for $\mu>1$ the distribution $P(\omega)$ is unimodal and centered at $\omega=1 / 2$. This signifies that in this case two searchers will most likely arrive at the target simultaneously.

Therefore, in situations when $0<\mu \leq 1$ (i.e. when $\Psi(\tau)$ does not already have the first moment) the first passage times of two searchers will be most probably distinctly 


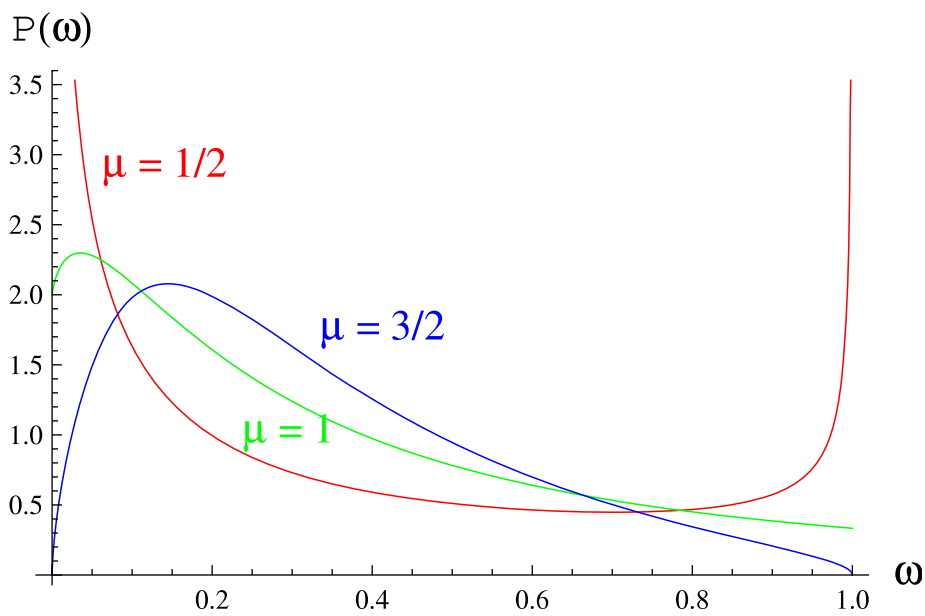

Figure 3. The distribution $P(\omega)$ in equation (22) for $\mu=1 / 2,1$ and $3 / 2$.

different. In contrast, for $\mu>1$ the first passage times will be most probably the same and hence the algorithm resulting in such values of $\mu$ will be robust.

We end this subsection by noticing that a similar transition was found in [49] in the related Sinai model with a linear drift of strength $\mu$. There the occupation time distribution on the positive axis exhibits a transition at $\mu=1$ and is also given, in certain limiting cases, by a beta distribution, as in equation (20).

\subsection{Three non-communicating random searchers}

For three non-communicating searchers, whose first passage times obey a non-truncated distribution in equation (4), we find

$P(\omega)=\frac{\sqrt{\pi}}{2^{4 \mu-1}} \frac{\Gamma(2 \mu) \Gamma(3 \mu)}{\Gamma^{3}(\mu) \Gamma(2 \mu+1 / 2)} \omega^{-1-\mu}(1-\omega)^{\mu-1}{ }_{2} F_{1}\left(2 \mu, 3 \mu ; 2 \mu+\frac{1}{2} ;-\frac{1-\omega}{4 \omega}\right)$,

where ${ }_{2} F_{1}$ is a hypergeometric series. The distribution in equation (22) for three different values of $\mu$ is depicted in figure 3 .

Consider first behavior of the distribution in equation (22) in the vicinity of $\omega=0$ and 1. Recollecting the definition of the hypergeometric series, one immediately observes from equation $(22)$ that $P(\omega) \sim C_{2}(1-\omega)^{\mu-1}$ when $\omega \rightarrow 1$. A little bit more involved analysis shows that $P(\omega) \sim C_{1} \omega^{\mu-1}$ when $\omega \rightarrow 0$. This means that, similarly to the $N=2$ case, the distribution $P(\omega)$ diverges at both edges when $\mu<1$ and the most probable values of $\omega$ are 0 and 1 . Note, however, that $C_{1}>C_{2}$ and hence the distribution is skewed to the left favoring small values of $\omega$. Therefore, for $\mu<1$ the most probable situation is that one of three searchers arrives at the location of the target much earlier than the other two. Clearly, the mean $\langle\omega\rangle=1 / 3$ does not have any significance (apart, of course, from the fact that this is just the first moment of the distribution in equation (22)).

Further on, the distribution in equation (22) exhibits a qualitative change of behavior for $\mu \geq 1$. Here $P(\omega)$ is always a bell-shaped function of the variable $\omega$ centered at the most probable value $\omega=\omega_{m}$. The only difference between the $\mu=1$ and $\mu>1$ cases is that for the former $P(\omega)$ attains a non-zero value at the edges, $P(\omega=1)=1 / 3$ and 


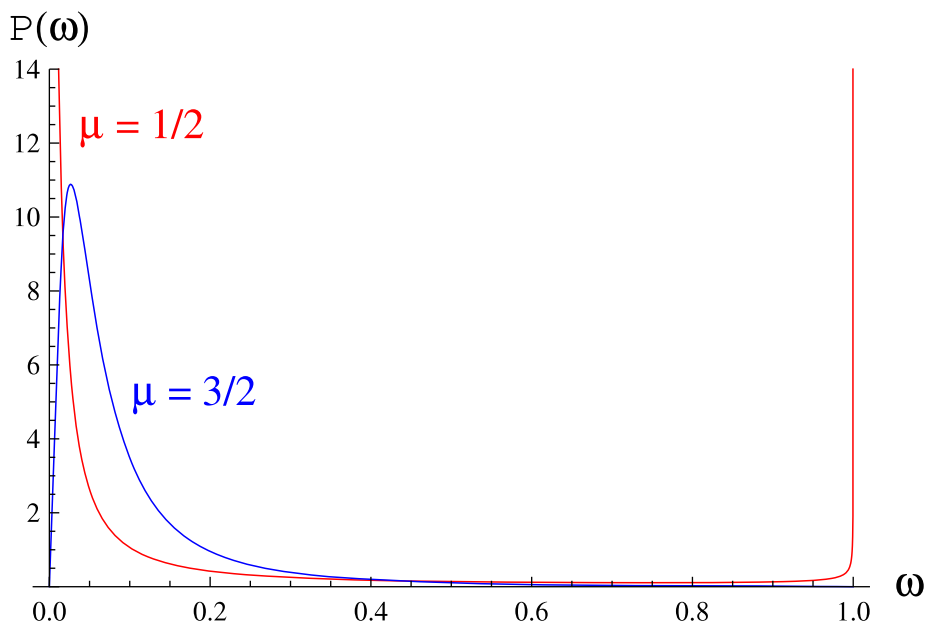

Figure 4. The distribution $P(\omega)$ in equations (23) (red line) and (24) (blue line) for $N=10$.

$P(\omega=0)=2$, while in the latter $P(\omega=0)=P(\omega=1) \equiv 0$. It is important to observe, however, that $\omega_{m}$ is always appreciably less than $\langle\omega\rangle=1 / 3$. For example, for $\mu=3$, one has $\omega_{m} \approx 0.2719$, for $\mu=10$ one has $\omega_{m} \approx 0.3102$, etc. In fact, $\omega_{m} \rightarrow 1 / 3$ only when $\mu \rightarrow \infty$.

\section{3. $N$ non-communicating random searchers for $\mu=1 / 2$ and $3 / 2$}

For $\mu=1 / 2$ and arbitrary $N$ (i.e. for $N$ BMs starting from the same point on a semiinfinite line), we find the following simple law:

$$
P(\omega)=\frac{N-1}{\pi} \frac{1}{\sqrt{\omega(1-\omega)}} \frac{1}{1-\omega+(N-1)^{2} \omega},
$$

while for $\mu=3 / 2$ and arbitrary $N$ we get

$$
\begin{aligned}
P(\omega)=\frac{\Gamma(N)}{\pi} & \frac{(1-\omega)^{1 / 2}}{\omega\left(1-\omega+(N-1)^{2} \omega\right)^{3 / 2}} \sum_{p=0}^{N-1} \frac{p+1}{\Gamma(N-p)} \\
& \times\left(\frac{\omega}{1-\omega+(N-1)^{2} \omega}\right)^{p / 2} \mathrm{U}_{p+1}\left(\left(\frac{\omega(N-1)^{2}}{1-\omega+(N-1)^{2} \omega}\right)^{1 / 2}\right),
\end{aligned}
$$

where $\mathrm{U}_{k}(\cdot)$ are the Chebyshev polynomials of the second kind. These distributions, for a particular case $N=10$, are depicted in figure 4 .

Note that, for $\mu=1 / 2$ and arbitrary $N$, the distribution $P(\omega)$ has a characteristic $U$ shape and diverges at the edges $\omega=0$ and 1 . The distribution function is strongly skewed toward the left edge; for $\omega \rightarrow 0$ the distribution behaves as $P(\omega) \sim(N-1) \omega^{-1 / 2}$, while for $\omega \rightarrow 1$ we have $P(\omega) \sim(N-1)^{-1}(1-\omega)^{-1 / 2}$, i.e. the amplitudes are $(N-1)^{2}$ times different. This means that for $N \gg 1$ an event in which one out of a swarm of $N$ independent searchers arrives at the target much earlier than the rest of the searchers is much more probable than an event in which one of the searchers arrives at the target location much later than the others. 
For $\mu=3 / 2$ the distribution $P(\omega)$ is unimodal and peaked at some value $\omega=\omega_{m}$, which defines the most probable first passage time of a given searcher relative to the ensemble-average value. Note, however, that $\omega_{m}$ is always less than the mean $\langle\omega\rangle=1 / N$. Actually, as one may observe from figure $4, \omega_{m}$ is more than two times less than 1/10. Note that in this case the mean first passage time for an individual searcher exists, but apparently is not a proper measure of the search process since it is different from the most probable value.

\section{Asymptotic large- $N$ behavior of $P(\omega)$ for heavy-tailed first passage time distributions}

We consider next the large- $N$ asymptotic behavior of the probability density $P(\omega)$ in equation (18). To do this, it might be helpful first to look at equation (18) from a different perspective and to realize that $P(\omega)$ can be expressed as the probability density function $P_{N-1}(|\mathbf{r}|)$ for the position of a random walker in some Rayleigh-type random flight model with a variable flight length in which $N$ - the number of independent searchers - will play the role of 'time'.

Consider a Rayleigh random flight process (see, e.g., [50]) in a $d=2 \mu$-dimensional continuum. If $d$ is thought to be an integer, this would restrict the analysis to some particular values of $\mu$. However, as noted in [51], formal considerations can be used to define an isotropic random flight in a space of arbitrary positive dimension $d$, with $d$ not necessarily restricted to integral values. This relaxes any constraint on the value of $\mu$.

Suppose that flight lengths are independent, identically distributed random variables and let the probability density function $p\left(\mathbf{r}, \mathbf{r}^{\prime}\right)$ for a transition from $\mathbf{r}$ to $\mathbf{r}^{\prime}$ be a function of $\rho=\left|\mathbf{r}-\mathbf{r}^{\prime}\right|$ only, which means that the process is translationally invariant and $p\left(\mathbf{r}, \mathbf{r}^{\prime}\right)=p(\rho)$. Choose next

$$
p(\rho)=\frac{\Gamma(2 \mu)}{\pi^{\mu} \Gamma(\mu)} \frac{1}{\left(1+\rho^{2}\right)^{2 \mu}},
$$

i.e. a heavy-tailed (but normalizable for any $\mu>0$ ) distribution.

Using Fourier transform technique (see, e.g., [50]), one readily finds that the probability density function of a random walker, starting at the origin, to be at a distance $\rho$ from the origin after $N-1$ such flights, is given by

$$
P_{N-1}(\rho)=\frac{1}{2^{\mu} \pi^{\mu} \rho^{\mu-1}} \int_{0}^{\infty} \mathrm{d} u u^{\mu} J_{\mu-1}(\rho u) \Xi^{N-1}(u),
$$

with $\Xi(u)$ defined by equation (19).

Consequently, we find the following relation between the probability density $P(\omega)$ in equation (18) and $P_{N-1}(\rho)$ in equation (26):

$$
\omega(1-\omega) P(\omega)=\left.\frac{\pi^{\mu}}{\Gamma(\mu)} \rho^{2 \mu} P_{N-1}(\rho)\right|_{\rho=\sqrt{(1-\omega) / \omega}} .
$$

Therefore, the probability density of the random variable $\omega$, which describes the realization-dependent ratio of the first passage time of a given searcher in an ensemble of $N$ ones, and of the ensemble-averaged first passage time, is proportional to the probability density of finding a random walker performing Rayleigh random flights with a broad 
distribution of flight length, equation (25), at a distance $\sqrt{(1-\omega) / \omega}$ away from the origin after $N-1$ flights.

When $N$ is large, the integral in equation (18) is dominated by the behavior of $\Xi(u)$ in the vicinity of $u=0$. In turn, the latter depends on the value $\mu$ : the cases $\mu>1, \mu=1$ and $\mu<1$ need to be considered separately.

\subsection{The case $\mu>1$}

In this case, the leading small- $u$ behavior of the characteristic function follows:

$$
\ln (\Xi(u)) \sim-u^{2} / 4(\mu-1) .
$$

Plugging the latter asymptotic form into equation (18), and performing integration over $\mathrm{d} u$, we find that the asymptotic large- $N$ behavior of the distribution $P(\omega)$ is determined by

$$
P(\omega) \sim \frac{1}{\omega(1-\omega)}\left(\frac{1-\omega}{\omega N}\right)^{\mu} \exp \left(-(\mu-1) \frac{(1-\omega)}{\omega N}\right) .
$$

Therefore, in the limit $N \gg 1$ for $\mu>1$ the distribution $P(\omega)$ is always a bell-shaped function of $\omega$, which approaches 0 exponentially fast when $\omega \rightarrow 0$ and as a power law when $\omega \rightarrow 1$. The maximum of $P(\omega)$ is located at

$$
\begin{aligned}
\omega_{m} & =\frac{1}{4 N}\left(\mu-1+(\mu+1) N-\sqrt{(\mu-1+(\mu+1) N)^{2}-8(\mu-1) N}\right) \\
& \sim \frac{\mu-1}{\mu+1} \frac{1}{N} .
\end{aligned}
$$

This substantiates our previous claims that $\omega_{m}$ is always less than $\langle\omega\rangle=1 / N$ and converges to $1 / N$ only when $\mu \rightarrow \infty$. Note that in this case the first moment of $\Psi(\tau)$, i.e. the mean first passage time, exists but it is not representative of the most probable behavior.

It is instructive now to reproduce the result in equation (29) using a different type of argument. Notice that $P_{N}(\rho)$ in equation (26) in the limit $N \rightarrow \infty$ becomes a Gaussian distribution in $(d=2 \mu)$-dimensional space of one rescaled variable $\rho / N^{1 / 2}$ :

$$
P_{N}(\rho) \sim \frac{1}{N^{\mu}} \exp \left(-(\mu-1) \frac{\rho^{2}}{N}\right) .
$$

Inserting the latter expression into the relation in equation (27), we recover the result in equation (29).

\subsection{The case $\mu=1$}

In this borderline case one finds

$$
\ln (\Xi(u)) \sim-(1-2 \gamma+2 \ln 2-2 \ln (u)) u^{2} / 4,
$$

where $\gamma$ is the Euler constant.

Note that here one has an additional logarithmic factor $\ln (u)$, as compared to the leading small- $u$ behavior in equation (28). Since a logarithm is a slowly varying function, 
we can repeat essentially the same argument: $P_{N}(\rho)$ in equation (26) converges, as $N \rightarrow \infty$, to a Gaussian distribution of the scaling variable $\rho /(N \log N)^{1 / 2}$ :

$$
P_{N}(\rho) \sim \frac{1}{N \ln (N)} \exp \left(-\frac{\rho^{2}}{N \ln (N)}\right) .
$$

Hence, by virtue of the relation in equation (27), we find

$$
P(\omega) \sim \frac{1}{(N \ln (N) \omega)^{2}} \exp \left(-\frac{(1-\omega)}{N \ln (N) \omega}\right) .
$$

This is again a bell-shaped function of $\omega$ with the most probable value $\omega_{m} \sim 1 /(2 N \log N)$.

\subsection{The case $\mu<1$}

For $\mu<1$ the leading small- $u$ behavior of the characteristic function is

$$
\ln (\Xi(u)) \sim-\frac{\Gamma(1-\mu)}{4^{\mu} \Gamma(1+\mu)} u^{2 \mu}
$$

so that for $N \rightarrow \infty, P_{N}(\rho)$ in equation (26) becomes

$$
P_{N}(\rho)=\frac{1}{N} S\left(x=\frac{\rho}{N^{1 / 2 \mu}}, \alpha=2 \mu, \sigma=\frac{\Gamma(1-\mu)}{\Gamma(1+\mu)}\right)
$$

where $S(\cdot)$ is the one-sided, $2 \mu$-dimensional stable law with index $\alpha=2 \mu$ and scale $\sigma=\Gamma(1-\mu) / \Gamma(1+\mu)[52]$. As a consequence, the distribution $P(\omega)$ is given, for $N \rightarrow \infty$, by

$$
P(\omega) \sim \frac{(1-\omega)^{\mu-1}}{N \omega^{\mu+1}} S\left(x=\frac{1}{N^{1 / 2 \mu}} \sqrt{\frac{1-\omega}{\omega}}, \alpha=2 \mu, \sigma=\frac{\Gamma(1-\mu)}{\Gamma(1+\mu)}\right) .
$$

Asymptotic behavior of $S(\cdot)$ has been discussed in detail in [52]. When $x \ll 1$, $S(\cdot) \rightarrow$ const and hence

$$
P(\omega) \sim \frac{(1-\omega)^{\mu-1}}{N}
$$

i.e. $P(\omega)$ diverges as $\omega \rightarrow 1$. On the other hand, when $x \gg 1$, i.e. when $\omega$ is sufficiently close to $0, S(\cdot) \sim 1 / x^{4 \mu}[52]$, which yields

$$
P(\omega) \sim N \omega^{\mu-1} .
$$

This means that $P(\omega)$ diverges when $\omega \rightarrow 0$ and, generally, in this domain $0<\mu<1$ the distribution has a characteristic U-shaped form strongly skewed towards small values of $\omega$ since the amplitudes differ by a factor $N^{2}$. Note that for $\mu=1 / 2$ the distribution in equation (37) becomes the Cauchy distribution so that the simple form in equation (23) follows immediately. 


\section{The distribution $P(\omega)$ for exponentially truncated first passage time distributions. Two non-communicating searchers}

We turn next to the analysis of the distribution $P(\omega)$ in the case when the parent first passage time distribution $\Psi(\tau)$, equation (6), possesses moments of arbitrary order.

For two non-communicating searchers and the exponentially truncated distribution in equation (6), we get the following result for the distribution of the random variable $\omega$ :

$$
P(\omega)=\frac{1}{2 K_{\mu}^{2}(2 \sqrt{a / b})} \frac{1}{\omega(1-\omega)} K_{2 \mu}\left(2 \sqrt{\frac{a}{b \omega(1-\omega)}}\right) .
$$

Two remarks are in order. First, one readily notices that $P(\omega)$ vanishes exponentially fast when $\omega \rightarrow 0$ or $\omega \rightarrow 1$ so that $P(\omega=0)=P(\omega=1)=0$. Second, $P(\omega)$ is clearly symmetric under the replacement $\omega \rightarrow 1-\omega$. Since here we deal with a truncated distribution $\Psi(\tau)$ which possesses the moments of arbitrary order, our first guess would be that $P(\omega)$ is always a bell-shaped function with a maximum at $\omega=1 / 2$. To check this guess, we expand $P(\omega)$ in the Taylor series around $\omega=1 / 2$ :

$$
\begin{aligned}
P(\omega)=\frac{2 K_{2 \mu}(4 \sqrt{a / b})}{K_{\mu}^{2}(2 \sqrt{a / b})}\left[1+4\left(1-\mu-2 \sqrt{\frac{a}{b}} \frac{K_{2 \mu-1}(4 \sqrt{a / b})}{K_{2 \mu}(4 \sqrt{a / b})}\right)\right. \\
\left.\times\left(\omega-\frac{1}{2}\right)^{2}+\mathcal{O}\left(\omega-\frac{1}{2}\right)^{4}\right] .
\end{aligned}
$$

Inspecting the sign of the coefficient before the quadratic term, i.e.

$$
g=1-\mu-2 \sqrt{\frac{a}{b}} \frac{K_{2 \mu-1}(4 \sqrt{a / b})}{K_{2 \mu}(4 \sqrt{a / b})},
$$

we notice that

- For $\mu>1, g$ is always negative for any value of $b / a$ so that here the distribution $P(\omega)$ is a bell-shaped function with a maximum at $\omega=1 / 2$.

- For $\mu=1, g$ is negative and approaches 0 from below when $b / a \rightarrow \infty$. It means that $P(\omega)$ is generally a bell-shaped function with a maximum at $\omega=1 / 2$, but it is becoming progressively flatter when $b / a$ is increased, so that ultimately $P(\omega) \approx 1$ apart from very narrow regions at the edges for $b / a \gg 1$.

- For $0 \leq \mu<1$ there always exists a critical value $y_{\mathrm{c}}(\mu)$ of the parameter $y=b / a$ which is defined implicitly as the solution of the equation $g=0$, equation (42). For $b / a<y_{\mathrm{c}}(\mu)$, the distribution $P(\omega)$ is unimodal with a maximum at $\omega=1 / 2$. For $b / a=y_{\mathrm{c}}(\mu)$, the distribution is nearly uniform except for narrow regions in the vicinity of the edges. Finally, which is quite surprising in view of the fact that in this case $\Psi(\tau)$ possesses all moments, for $b / a>y_{\mathrm{c}}(\mu)$ the distribution $P(\omega)$ is bimodal with a characteristic M-shaped form, two maxima close to 0 and 1 and $\omega=1 / 2$ being the least probable value.

We depict in figure 5 three characteristic forms of $P(\omega)$ for $\mu=1 / 2$ and three different values of $b / a$.

Therefore, for $\mu \geq 1$ two non-communicating searchers will most probably reach the target simultaneously. For $0 \leq \mu<1$ two distinctly different situations are possible: if 


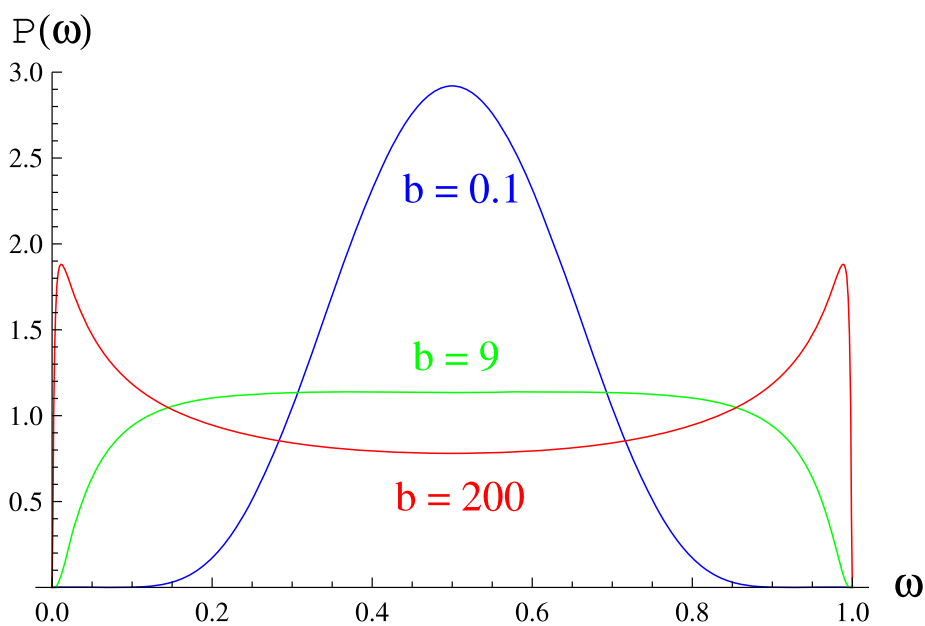

Figure 5. The distribution $P(\omega)$ in equation (40) for $\mu=1 / 2$ and different values of $b$ ( $a$ is set equal to 1$)$.

$b / a$ is less than some well-defined critical value $y_{\mathrm{c}}(\mu)$, then most likely both searchers will arrive at the target for the first time together. If, in contrast, $b / a$ exceeds this critical value, the event in which both searchers arrive at the location of the target simultaneously will be the least probable one.

\subsection{Two independent BMs in a semi-infinite $1 D$ system with a bias}

Recall now that the distribution in equation (6) with $\mu=1 / 2$ is exact for a BM in semiinfinite 1D systems in the presence of a constant bias pointing towards the target. In this case one has $a=x_{0}^{2} / 4 D$, where $x_{0}$ is the starting point, $D$ is the diffusion coefficient and $b=4 D / v^{2}, v$ being the drift velocity. Hence, $2 \sqrt{a / b}=P e=x_{0}|v| / 2 D$ is the Peclet number (see, e.g., [33]). Consequently, we can make the following statement:

Consider two independent, absolutely identical BMs on a semi-infinite line, starting at the same point $x_{0}$, having the same diffusion coefficient $D$ and experiencing the same bias $F$ which points towards the origin so that the drift velocity of both BMs is $v<0$. Then, an event in which both BMs arrive for the first time to the origin simultaneously is

- the least probable if $P e<P e_{\mathrm{c}}$,

- the most probable if $P e>P e_{\mathrm{c}}$,

where $P e_{\mathrm{c}}$ is the solution of the transcendental equation:

$$
1=2 P e_{\mathrm{c}} \frac{K_{0}\left(2 P e_{\mathrm{c}}\right)}{K_{1}\left(2 P e_{\mathrm{c}}\right)}
$$

An approximate solution of the latter equation gives $P e_{\mathrm{c}} \approx 0.666 \cdots$.

Therefore, the mean first passage time to the target might be an appropriate measure of the search efficiency for sufficiently large Peclet numbers, but definitely is not the one in the case of small $P e$. In the latter case the sample-to-sample fluctuations are significant and the mean value is not representative of the actual behavior. 
First passages for a search by a swarm of independent random searchers

\subsection{Two independent unbiased $\mathrm{BMs}$ on a finite interval}

We have already remarked that the expression in equation (6) is a reasonable approximation for the first passage time distribution for random motion in finite systems. For a one-dimensional bounded interval of length $L$ and, for $\mu=1 / 2$, the parameter $b \sim L^{2} / D$, while $a \sim x_{0}^{2} / D$, where $x_{0}$ is the starting point. Consequently, $\sqrt{a / b}$ should be $\sim x_{0} / L$ and independent of the particles' diffusion coefficient $D$. This suggests a somewhat strange result that the very shape (or the modality) of the distribution $P(\omega)$ will crucially depend on the starting point $x_{0}$.

To verify such a prediction, we focus now on a BM on a finite $1 \mathrm{D}$ interval and reexamine this question using an exact form of the normalized first passage time distribution $\Psi(\tau)$. For the BM with a starting point at $x_{0}$ on an interval of length $L$ with an adsorbing boundary at $x=0$ and a reflecting boundary at $x=L, \Psi(\tau)$ is given by

$$
\Psi(\tau)=\frac{2 \pi D}{L^{2}} \sum_{n=0}^{\infty} A_{n}^{(d=1)}\left(\frac{x_{0}}{L}\right) \exp \left(-\frac{\pi^{2}(n+1 / 2)^{2} D \tau}{L^{2}}\right),
$$

where

$$
A_{n}^{(d=1)}\left(\frac{x_{0}}{L}\right)=\left(n+\frac{1}{2}\right) \sin \left(\frac{\pi(n+1 / 2) x_{0}}{L}\right) .
$$

Consequently, the normalized distribution $P(\omega)$ in the case of two independent, identical BMs has the following form:

$$
P(\omega)=\frac{4}{\pi^{2}} \sum_{m, n=0}^{\infty} \frac{A_{n}^{(d=1)}\left(x_{0} / L\right) A_{m}^{(d=1)}\left(x_{0} / L\right)}{\left(\omega(n+1 / 2)^{2}+(1-\omega)(m+1 / 2)^{2}\right)^{2}} .
$$

Using next an equality

$$
\frac{1}{\lambda_{m}^{2}} \frac{\mathrm{d}}{\mathrm{d} \omega} \frac{1}{\lambda_{n}^{2}+((1-\omega) / \omega) \lambda_{m}^{2}}=\frac{1}{\left(\omega \lambda_{n}^{2}+(1-\omega) \lambda_{m}^{2}\right)^{2}},
$$

and the following representation of the characteristic function $\Phi(\lambda)$ of the distribution $\Psi(\tau)$ in equation (44):

$$
\begin{aligned}
\Phi(\lambda) & =\int_{0}^{\infty} \mathrm{d} \tau \Psi(\tau) \exp (-\lambda \tau) \\
& =\frac{2}{\pi} \sum_{n=0}^{\infty} \frac{A_{n}^{(d=1)}\left(x_{0} / L\right)}{(n+1 / 2)^{2}+L^{2} \lambda / \pi^{2} D} \\
& =\frac{\cosh \left(\left(L-x_{0}\right) \sqrt{\lambda / D}\right)}{\cosh (L \sqrt{\lambda / D})},
\end{aligned}
$$

we can conveniently rewrite equation (46) as

$$
\begin{aligned}
P(\omega)=\frac{2}{\pi} \frac{\mathrm{d}}{\mathrm{d} \omega} & \sum_{m=0}^{\infty} \frac{\sin \left(\pi(m+1 / 2)\left(x_{0} / L\right)\right)}{m+1 / 2} \Phi\left(\lambda=\frac{\pi^{2} D}{L^{2}} \frac{1-\omega}{\omega}(m+1 / 2)^{2}\right) \\
= & \frac{2}{\pi} \frac{\mathrm{d}}{\mathrm{d} \omega} \sum_{m=0}^{\infty} \frac{\sin \left(\pi(m+1 / 2)\left(x_{0} / L\right)\right)}{m+1 / 2} \\
& \times \frac{\cosh \left(\pi(m+1 / 2) \sqrt{(1-\omega) / \omega}\left(1-\left(x_{0} / L\right)\right)\right)}{\cosh (\pi(m+1 / 2) \sqrt{(1-\omega) / \omega})} .
\end{aligned}
$$




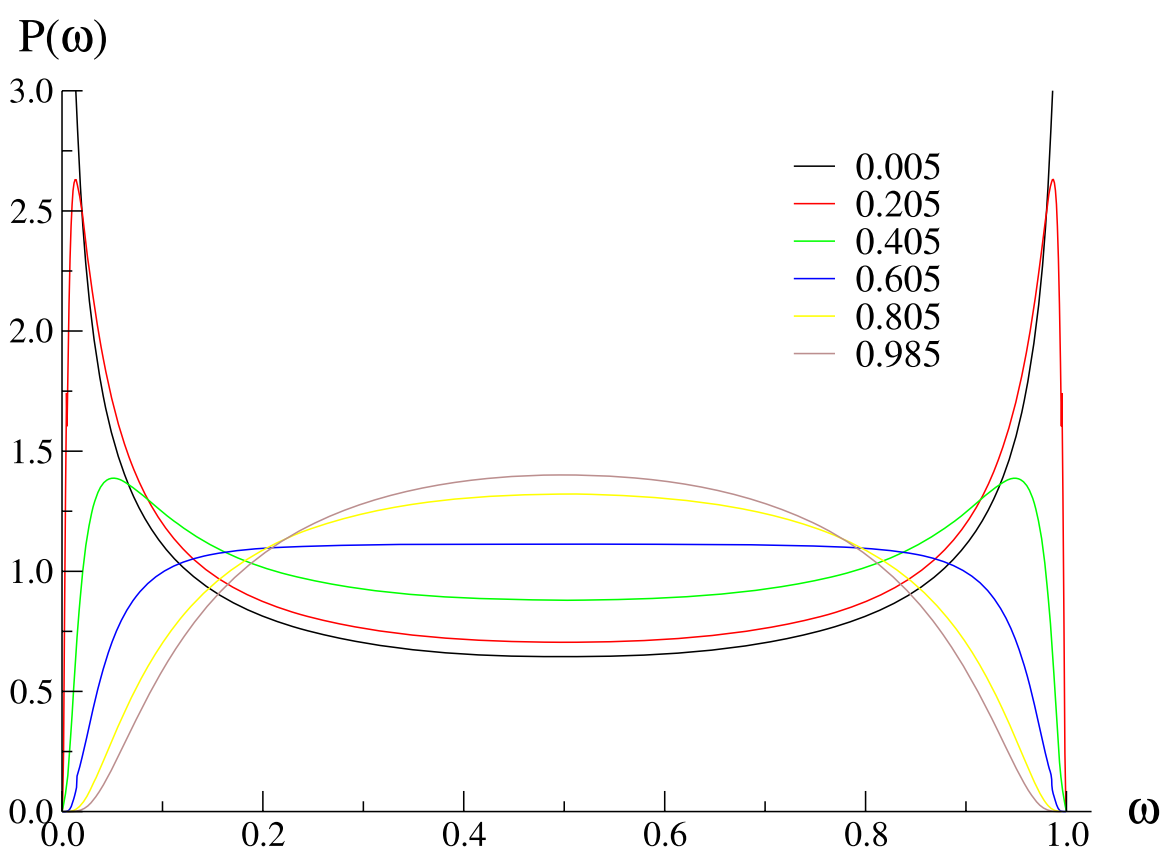

Figure 6. Two BMs on a finite interval $[0, L]$ with a reflecting boundary at $x=L$. The distribution $P(\omega)$ in equation (49) for different values of $x_{0} / L, x_{0}$ being the starting point of both BMs.

The distribution $P(\omega)$ tends to zero exponentially, $P(\omega) \sim \exp \left(-\pi x_{0} / 2 L \sqrt{\omega}\right) / \omega^{3 / 2}$, when $\omega \rightarrow 0$, precisely in the same way as $P(\omega)$ in equation (40) obtained for the exponentially truncated first passage time distribution. By symmetry, we expect the same behavior when $\omega \rightarrow 1$. In principle, $P(\omega)$ in equation (49) can be represented in closed form as a complicated combination of elliptic function. However, we prefer to proceed with a numerical analysis of the rapidly convergent series in equation (49) in order to understand whether $P(\omega)$ is always a bell-shaped function of $\omega$ or undergoes a transition to an M-shaped form at a certain value of $x_{0} / L$.

In figure 6 we depict the distribution $P(\omega)$ in equation (49) for different values of the ratio $x_{0} / L$. One notices that $P(\omega)$ has a different shape depending whether $x_{0} / L$ is less than or greater than the critical value $\approx 0.605 \cdots$. This allows us to make the following statement.

Consider two independent, identical, unbiased BMs starting at the same point $x_{0}$ on a finite interval $[0, L]$ with a reflecting boundary at $x=L$. Then, an event in which both BMs arrive simultaneously at the origin is

- the least probable if $x_{0} / L \lesssim 0.605 \cdots$,

- the most probable if $x_{0} / L \gtrsim 0.605 \cdots$.

From a commonsense point of view such a behavior seems a bit counterintuitive indeed, why should two BMs arrive at the target at progressively distinct times the closer they are to its location, and should most probably arrive together when they are far from it? On the other hand, such a behavior is quite a natural one: indeed, $x_{0}\left(a^{1 / 2}\right)$ and $L\left(b^{1 / 2}\right)$ define the effective size of the window in which the decay of the first 


\section{$\log \Psi(\tau)$}

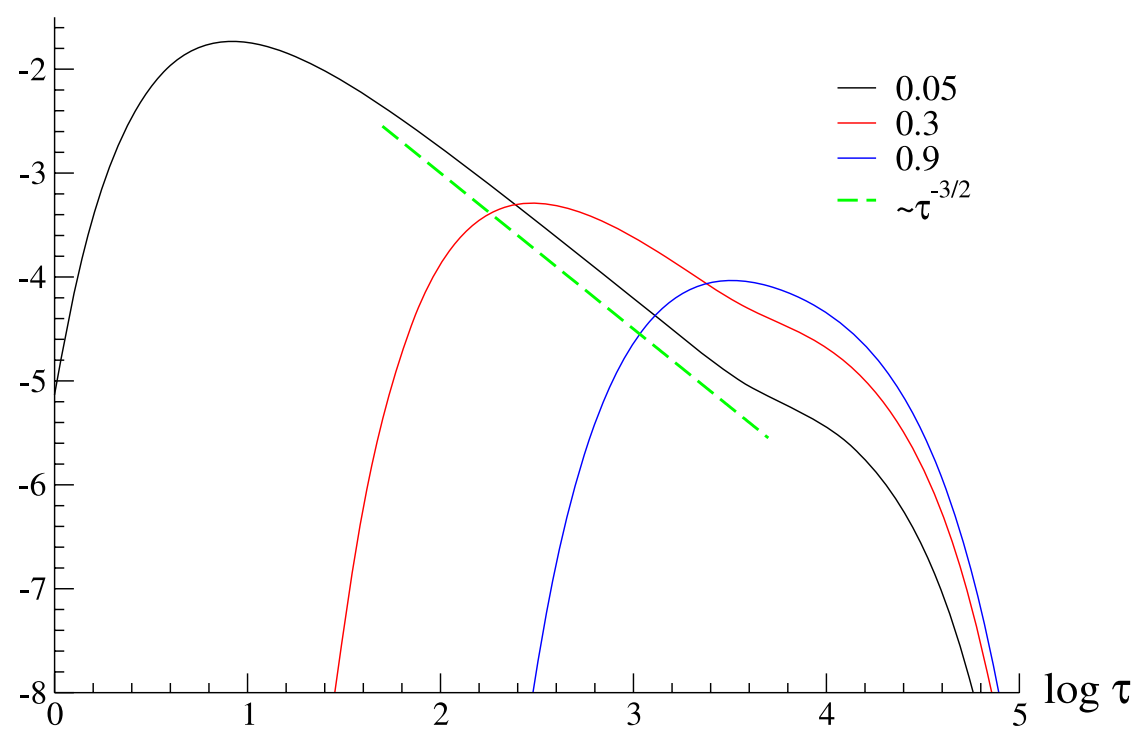

Figure 7. A BM on a finite interval $[0, L]$ (with a reflecting boundary at $x=L$ ) starting at $x=x_{0}$. The first passage time distribution $\Psi(\tau)$ in equation (44) for $L=100, D=1 / 2$ and different values of $x_{0} / L$.

passage time distribution is governed by the intermediate power-law tail. The larger this window is, the closer we are to the situation described in section 3. Therefore, the origin of such a disproportionate behavior of two identical, independent BMs is precisely the same as the one behind the famous arcsine law for the distribution of the fraction of time spent by a random walker on a positive half-axis [53]: once one of the BMs goes away from the target, it finds it more difficult to return than to keep on going away.

To substantiate this claim, we plot in figure 7 the distribution in equation (44) for three different values of $x_{0}$ and fixed $L$. Note that all three curves show an exponential behavior for both small and large values of $\tau$ (which, in fact, is an argument in favor of our choice of the exponentially truncated distribution in equation (6)). For large $\tau$ all three curves merge which signifies that at such values of $\tau$ the characteristic decay time is dependent only on $L$. The lower cutoff is clearly dependent only on the starting point $x_{0}$. Further on, we notice that the closer (for a fixed $L$ ) the starting point $x_{0}$ is to the reflecting boundary, the narrower is the distribution $\Psi(\tau)$. In contrast, the smaller $x_{0}$ is (the closer to the target), the more pronounced the intermediate power-law behavior $\sim t^{-3 / 2}$ becomes (see the dashed line in figure 7). Actually, the fact that the further away the starting point is from the reflecting wall, the broader is the first passage time distribution (the BM simply does not 'know' that it is in a finite system up to times of order $\sim L^{2} / D$ ) has been already discussed in detail in [33].

Consider next some standard characteristics of the first passage time distribution in equation (44), such as the mean first passage time, the skewness, the coefficient of variation and the kurtosis, to see if any of them reflects the transition observed for $P(\omega)$ at $x_{0} / L \approx 0.605 \cdots$. Note that the moments of arbitrary order of the distribution 
$\Psi(\tau)$ in equation (44) can be straightforwardly calculated in closed form:

$$
\left\langle\tau^{m}\right\rangle \equiv \int_{0}^{\infty} \tau^{m} \mathrm{~d} \tau \Psi(\tau)=\frac{(-1)^{m} \sqrt{\pi}}{\Gamma(m+1 / 2)}\left(\frac{L^{2}}{D}\right)^{m} E_{2 m}\left(\frac{x_{0}}{2 L}\right),
$$

where $E_{2 k}(\cdot)$ are the Euler polynomials. Consequently, the mean and the variance are given by

$$
\langle\tau\rangle=-2 \frac{L^{2}}{D} E_{2}\left(\frac{x_{0}}{2 L}\right)
$$

and

$$
k_{2}=\left\langle\tau^{2}\right\rangle-\langle\tau\rangle^{2}=\frac{4}{3} \frac{L^{4}}{D^{2}}\left(E_{4}\left(\frac{x_{0}}{2 L}\right)-3 E_{2}^{2}\left(\frac{x_{0}}{2 L}\right)\right)
$$

respectively. One can readily check that both characteristic properties are monotonically increasing functions of $x_{0} / L$ and do not show any sign of a particular behavior at $x_{0} / L \approx 0.605 \cdots$. Further on, we define the coefficient of variation

$$
\gamma_{v}=\sqrt{\frac{k_{2}^{2}}{\langle\tau\rangle^{2}}}=\sqrt{\frac{E_{4}\left(x_{0} / 2 L\right)-3 E_{2}^{2}\left(x_{0} / 2 L\right)}{3 E_{2}^{2}\left(x_{0} / 2 L\right)}},
$$

the skewness

$$
\gamma_{a}=\frac{k_{3}}{k_{2}^{3 / 2}}
$$

where $k_{3}$ is the third cumulant:

$$
k_{3}=\left\langle\tau^{3}\right\rangle-3\langle\tau\rangle\left\langle\tau^{2}\right\rangle+2\langle\tau\rangle^{3},
$$

and the kurtosis (coefficient of excess)

$$
\gamma_{e}=\frac{k_{4}}{k_{2}^{2}}
$$

with $k_{4}$ being the fourth cumulant of the distribution in equation (44):

$$
k_{4}=\left\langle\tau^{4}\right\rangle-3\left\langle\tau^{2}\right\rangle^{2}-4\langle\tau\rangle\left\langle\tau^{3}\right\rangle+12\langle\tau\rangle^{2}\left\langle\tau^{2}\right\rangle-6\langle\tau\rangle^{4} .
$$

In figure 8 we plot the coefficient of variation, the skewness and the kurtosis of the distribution in equation (44). One observes a strong variation of these properties reflecting an influence of extreme events for sufficiently small values of $x_{0} / L$. For $x_{0} / L>0.6$ the variation of $\gamma_{a}$ and $\gamma_{e}$ becomes rather small. However, neither of these properties shows a clear demarcation line between different regimes exhibited by $P(\omega)$.

We finally remark that in many practically interesting physical problems the starting point $x_{0}$ is not fixed, but the searcher rather starts from some random location which is uniformly distributed on the interval. The distribution $P_{\mathrm{av}}(\omega)$ appropriate to such a situation is obtained by merely averaging $P(\omega)$ in equation (49) over $x_{0}$, i.e.

$$
P_{\mathrm{av}}(\omega)=\frac{1}{L} \int_{0}^{L} \mathrm{~d} x_{0} P(\omega)
$$

A quick inspection of $P(\omega)$ in equation (49) shows that, due to the orthogonality of $A_{n}^{(d=1)}\left(x_{0} / L\right)$, the averaged distribution $P_{\mathrm{av}}(\omega)$ does not depend on $\omega$, so that

$$
P_{\mathrm{av}}(\omega) \equiv 1 \text {. }
$$

This seems to be a general property of $P_{\mathrm{av}}(\omega)$, associated with the probability conservation and thus, as will be checked for further examples, is independent of the dimension of space. 


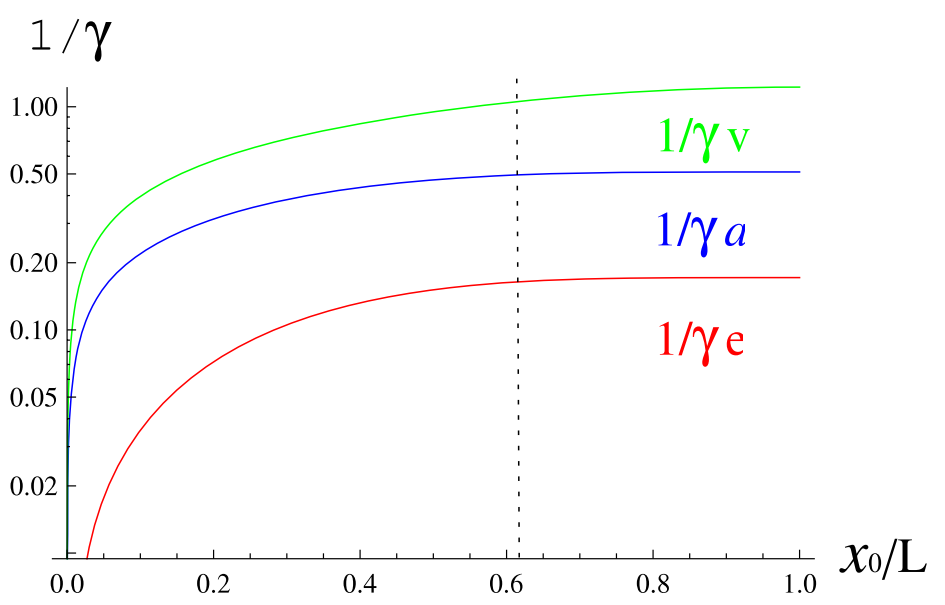

Figure 8. The coefficient of variation $\gamma_{v}$, the skewness $\gamma_{a}$ and the kurtosis $\gamma_{e}$ of the distribution $\Psi(\tau)$ in equation (44) versus $x_{0} / L$. Dotted vertical line defines the critical value $x_{0} / L \approx 0.605 \cdots$ at which the distribution $P(\omega)$ changes the modality.

\subsection{Two independent BMs in a disc with a reflecting boundary}

Recall that in an infinite 2D system the first passage time distribution has an algebraic (with a logarithmic correction) tail with $\mu=0$, equation (5). Consequently, we may expect essentially the same behavior as we observed in two previous subsections.

Consider, as depicted in figure 9, an immobile target of radius $r$ fixed at the origin of a disc of radius $L$ with a reflecting boundary. Suppose next that a BM starts at some point at distance $x_{0}$ from the origin and hits the target for the first time at time moment $\tau$. Then, the first passage time distribution $\Psi(\tau)$ in such a situation is given explicitly by

$$
\Psi(\tau)=\frac{r D}{Z} \sum_{n=0}^{\infty} A_{n}^{(d=2)}\left(r, x_{0}, L\right) \exp \left(-\lambda_{n}^{2} D \tau\right),
$$

where $Z$ is the normalization:

$$
\begin{aligned}
& A_{n}^{(d=2)}\left(r, x_{0}, L\right)=\frac{U_{0}\left(\lambda_{n} x_{0}\right) U_{0}^{\prime}\left(\lambda_{n} r\right)}{\lambda_{n}^{2} L^{2} U_{0}^{2}\left(\lambda_{n} L\right)-r^{2}\left(U_{0}^{\prime}\left(\lambda_{n} r\right)\right)^{2}}, \\
& U_{0}(x)=Y_{0}\left(\lambda_{n} r\right) J_{0}(x)-J_{0}\left(\lambda_{n} r\right) Y_{0}(x), \\
& U_{0}^{\prime}\left(\lambda_{n} r\right)=\left.\frac{\mathrm{d} U_{0}\left(\lambda_{n} x\right)}{\mathrm{d} x}\right|_{x=r},
\end{aligned}
$$

while $\lambda_{n}$ are the roots of the function

$$
Y_{0}\left(\lambda_{n} r\right) J_{1}\left(\lambda_{n} L\right)-J_{0}\left(\lambda_{n} r\right) Y_{1}\left(\lambda_{n} L\right)
$$

arranged in an ascending order, and $Y_{n}(\cdot)$ are Bessel functions of the second kind. Note that $\lambda_{n}$ depends on $L$ and $r$. 


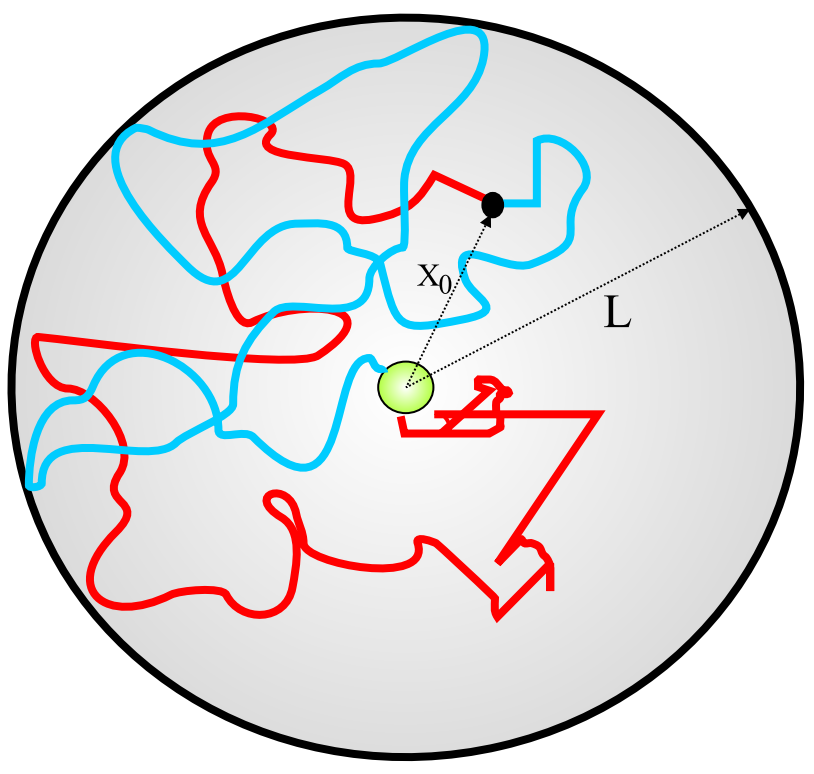

Figure 9. Two BMs in a (2D or 3D) bounded spherical domain of radius $L$ with a reflecting (thick black line) boundary. The target is a circle (in $3 \mathrm{D}$, a sphere) of radius $r$ centered at the origin. Two BMs start from the same point (a filled circle) at a distance $x_{0}$ from the center and arrive at the target boundary for the first time at time moments $\tau_{1}$ and $\tau_{2}$, respectively.

In figure 10 we depict the distribution in equation (60) for fixed $L, D, r$ and several values of $x_{0}$. Note that, apart from the intermediate behavior, which follows a power law with a logarithmic correction $\sim 1 / \tau \log ^{2}(\tau)$ (see the dashed line in figure 10), we have here essentially the same trend as the one we observed for a BM on a 1D finite interval. Namely, the closer the starting point is to the location of the target, the broader is the first passage time distribution so that the intermediate power-law behavior gets more pronounced.

Suppose next that we have two BMs starting at the same point some distance $x_{0}$ apart from the origin. Then, from equation (60), we get the following result for the distribution $P(\omega)$ :

$$
P(\omega)=\frac{r^{2}}{Z^{2}} \sum_{n, m=0}^{\infty} \frac{A_{n}^{(d=2)}\left(r, x_{0}, L\right) A_{m}^{(d=2)}\left(r, x_{0}, L\right)}{\left(\omega \lambda_{n}^{2}+(1-\omega) \lambda_{m}^{2}\right)^{2}}
$$

which can be conveniently rewritten, using the equality in equation (47), as

$$
P(\omega)=\frac{r}{Z} \frac{\mathrm{d}}{\mathrm{d} \omega} \sum_{m=0}^{\infty} \frac{A_{m}^{(d=2)}\left(r, x_{0}, L\right)}{\lambda_{m}^{2}} \Phi\left(\lambda=\frac{1-\omega}{\omega} D \lambda_{m}^{2}\right),
$$

where $\Phi(\lambda)$ is the characteristic function of the first passage time distribution in equation (60) defined by [33]

$\Phi(\lambda)=\frac{I_{0}\left(\sqrt{\lambda / D} x_{0}\right) K_{1}(\sqrt{\lambda / D} L)+K_{0}\left(\sqrt{\lambda / D} x_{0}\right) I_{1}(\sqrt{\lambda / D} L)}{I_{0}(\sqrt{\lambda / D} r) K_{1}(\sqrt{\lambda / D} L)+K_{0}(\sqrt{\lambda / D} r) I_{1}(\sqrt{\lambda / D} L)}$. 
$\log \Psi(\tau)$

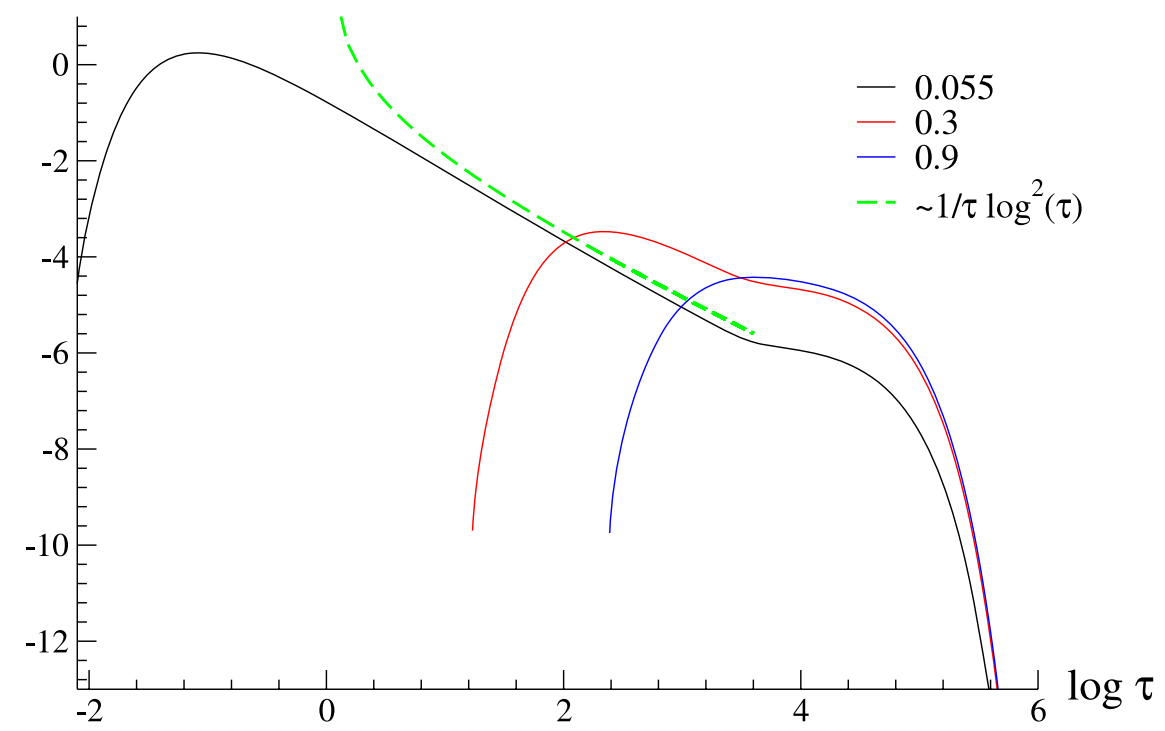

Figure 10. A BM in a two-dimensional disc of radius $L$ with a reflecting boundary. First passage time distribution $\Psi(\tau)$ in equation (60) for $D=1 / 2, L=100, r=5$ and different values of $x_{0} / L$.

We depict in figure 11 the result in equation (66) for fixed $L$ and $r$, and several values of $x_{0} / L$. One notices that the shape of the distribution $P(\omega)$ is again different, depending on the value of the ratio $x_{0} / L$. For $x_{0} / L \lesssim 0.643$, the distribution has an M-shaped form with a minimum at $\omega=1 / 2$, for $x_{0} / L \approx 0.643$ the distribution is nearly uniform except for narrow regions near the edges, and, finally, for $x_{0} / L \gtrsim 0.643$ it becomes unimodal, but still it is very broad and has a pronounced flat region around the maximum. This signifies that even in this regime the sample-to-sample fluctuations are significant.

Consider finally the situation when the starting point of two BM is uniformly distributed within the space between two concentric circles of radius $r$ and radius $L$. One readily finds, by averaging the result in equation (66), that here as in 1D one has $P_{\mathrm{av}}(\omega) \equiv 1$.

\subsection{Two independent BMs in a sphere with a reflecting boundary}

We now turn our attention to a Brownian motion in a 3D spherical domain with a reflecting boundary. The target is supposed to be a sphere of radius $r$ which is fixed at the origin.

The distribution of the first passage time $\tau$ of a BM starting at a distance $x_{0}$ from the origin to the surface of the target is given explicitly by

$$
\Psi(\tau)=\frac{D}{Z} \sum_{n=0}^{\infty} A_{n}^{(d=3)}\left(r, x_{0}, L\right) \exp \left(-\lambda_{n}^{2} D \tau\right),
$$

where

$$
\begin{aligned}
& A_{n}^{(d=3)}\left(r, x_{0}, L\right)=\frac{2 u_{0}\left(\lambda_{n} x_{0}\right) u_{0}^{\prime}\left(\lambda_{n} r\right)}{G\left(r, L, \lambda_{n}\right)}, \\
& u_{0}(x)=y_{0}\left(\lambda_{n} r\right) j_{0}(x)-j_{0}\left(\lambda_{n} r\right) y_{0}(x),
\end{aligned}
$$




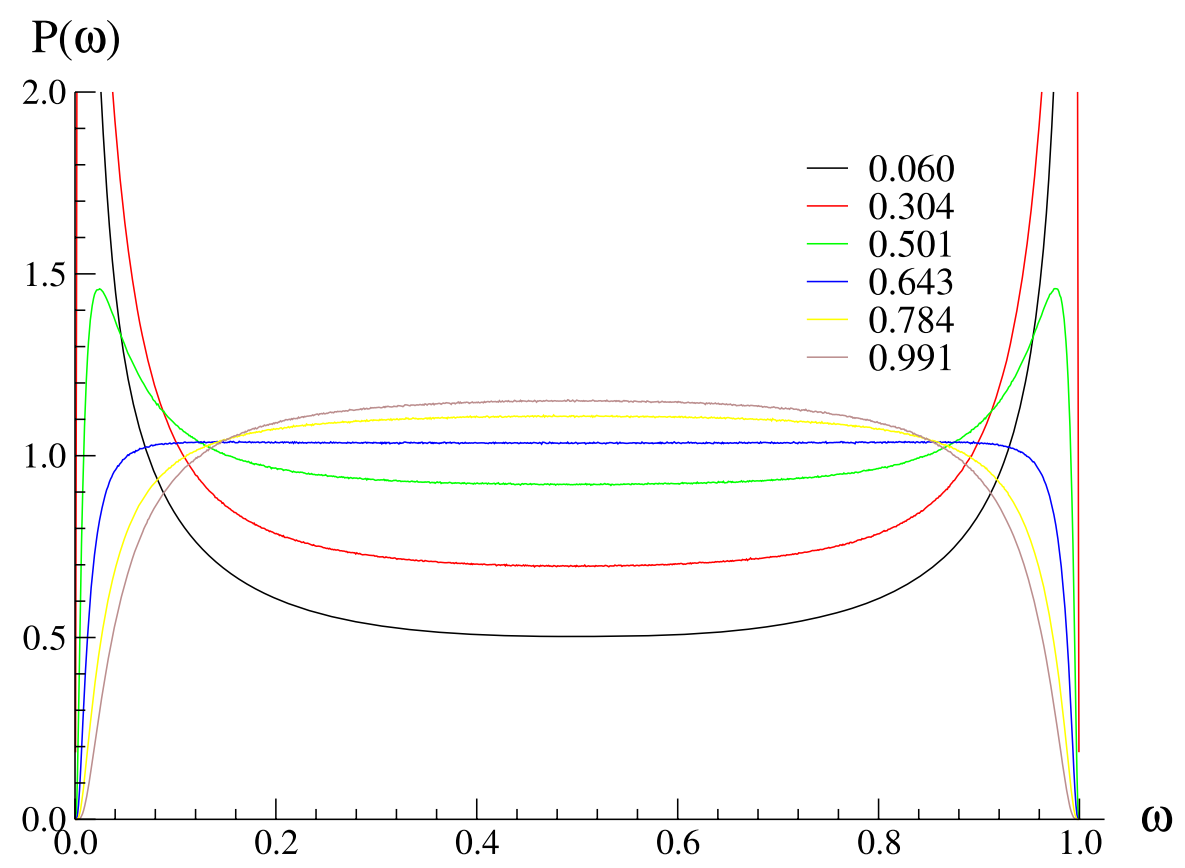

Figure 11. Two Brownian motions in a two-dimensional disc. The distribution $P(\omega)$ in equation (66) for $L=100, r=5$ and different values of $x_{0} / L$.

the derivative $u_{0}^{\prime}\left(\lambda_{n} r\right)=\mathrm{d} u_{0}\left(\lambda_{n} x\right) /\left.\mathrm{d} x\right|_{x=r}$ and

$G\left(r, L, \lambda_{n}\right)=L j_{0}\left(\lambda_{n} L\right) u_{0}\left(\lambda_{n} L\right)\left(\lambda_{n} L j_{0}\left(\lambda_{n} r\right)-y_{0}\left(\lambda_{n} r\right)\right)-\frac{j_{0}\left(\lambda_{n} r\right) y_{0}\left(\lambda_{n} r\right)}{\lambda_{n}}+\frac{L-r}{r^{2} \lambda_{n}^{4}}$.

The set $\left\{\lambda_{n}\right\}$ are the roots of $y_{0}\left(\lambda_{n} r\right) j_{1}\left(\lambda_{n} L\right)-j_{0}\left(\lambda_{n} r\right) y_{1}\left(\lambda_{n} L\right)$, arranged in an ascending order, while $j_{n}(\cdot)$ and $y_{n}(\cdot)$ are the spherical Bessel functions of the first and second kind, respectively.

The distribution of the first passage time in equation (60) is plotted in figure 12 for three different values of $x_{0} / L$. An intermediate power-law $\sim \tau^{-3 / 2}$ is apparent for $x_{0} / L=0.055$, persists for about a decade for $x_{0} / L=0.3$ and is entirely absent for $x_{0} / L=0.9$, i.e. when the BM starts close to the reflecting boundary.

From equation (68), we obtain the normalized distribution $P(\omega)$ for two independent BMs starting at a distance $x_{0}$ from the origin:

$$
\begin{aligned}
P(\omega) & =\frac{1}{Z^{2}} \sum_{n, m=0}^{\infty} \frac{A_{n}^{(d=3)}\left(r, x_{0}, L\right) A_{m}^{(d=3)}\left(r, x_{0}, L\right)}{\left(\omega \lambda_{n}^{2}+(1-\omega) \lambda_{m}^{2}\right)^{2}} \\
& =\frac{1}{Z} \sum_{m=0}^{\infty} \frac{A_{m}^{(d=3)}\left(r, x_{0}, L\right)}{\lambda_{m}^{2}} \Phi\left(\lambda=\frac{1-\omega}{\omega} D \lambda_{m}^{2}\right),
\end{aligned}
$$

where $\Phi(\lambda)$ is the characteristic function of $\Psi(\tau)$ in equation (68), defined by [33]

$\Phi(\lambda)=\frac{r}{x_{0}} \frac{\sinh \left(\sqrt{\lambda / D}\left(L-x_{0}\right)\right)-\sqrt{\lambda / D} L \cosh \left(\sqrt{\lambda / D}\left(L-x_{0}\right)\right)}{\sinh (\sqrt{\lambda / D}(L-r))-\sqrt{\lambda / D} L \cosh (\sqrt{\lambda / D}(L-r))}$. 


\section{$\log \Psi(\tau)$}

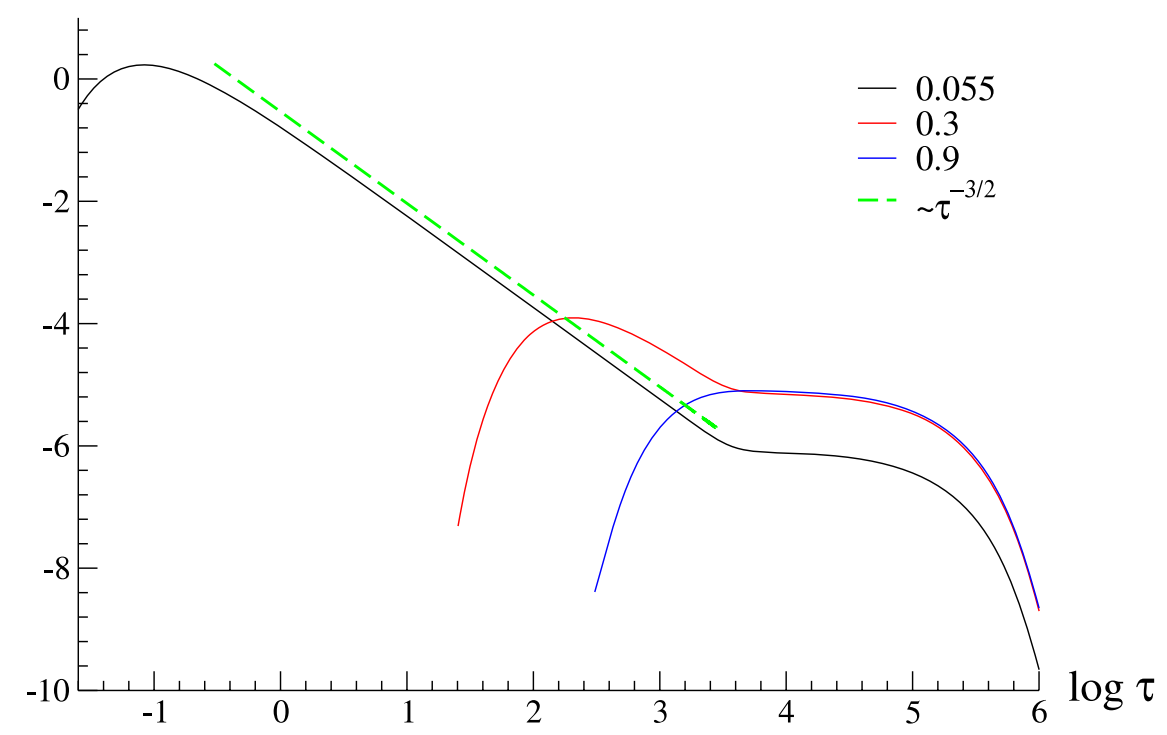

Figure 12. A BM in a sphere with a reflecting boundary. First passage time distribution $\Psi(\tau)$ in equation (68) for $L=100, r=5, D=1 / 2$ and different values of $x_{0} / L$.

This distribution is plotted in figure 13 for fixed $L$ and $r$, and several values of $x_{0} / L$. Therefore, also in 3D we find that $P(\omega)$ has a different modality depending on the value of the ratio $x_{0} / L$. For $x_{0} / L \lesssim 0.68 \cdots$, the distribution has an M-shaped form with $\omega=1 / 2$ being the least probable value. For $x_{0} / L>0.68 \cdots$, the distribution has, in principle, a maximum at $\omega=1 / 2$ but this maximum is almost invisible so that visually the distribution looks more like a uniform one, as compared to the $1 \mathrm{D}$ case in which the maximum is more apparent.

In the case when the starting point $x_{0}$ is uniformly distributed between two concentric spheres of radii $r$ and $L$, we again find $P_{\mathrm{av}}(\omega) \equiv 1$.

\subsection{Two independent BMs in a sphere with an adsorbing boundary}

Consider finally a geometrically different situation in which two BMs start from the same point within a three-dimensional sphere at a fixed distance $x_{0}$ from the origin but now the target is the surface of the sphere. In this case, the normalized distribution of the time of the first passage of a BM with diffusion coefficient $D$ to any point on the surface of the sphere of radius $L$ from a point at distance $x_{0}$ from the origin is given by the series (see, e.g., $[6,54])$

$$
\Psi(\tau)=\frac{2 \pi D}{x_{0} L} \sum_{n=1}^{\infty}(-1)^{n+1} n \sin \left(\pi n \frac{x_{0}}{L}\right) \exp \left(-\frac{\pi^{2} n^{2} D \tau}{L^{2}}\right),
$$

whose moments of arbitrary order $m$ are defined as

$$
\left\langle\tau^{m}\right\rangle=\frac{(-1)^{m} \sqrt{\pi}}{\Gamma(m+3 / 2)} \frac{L}{x_{0}}\left(\frac{L^{2}}{D}\right)^{m} B_{2 m+1}\left(\frac{x_{0}+L}{2 L}\right),
$$




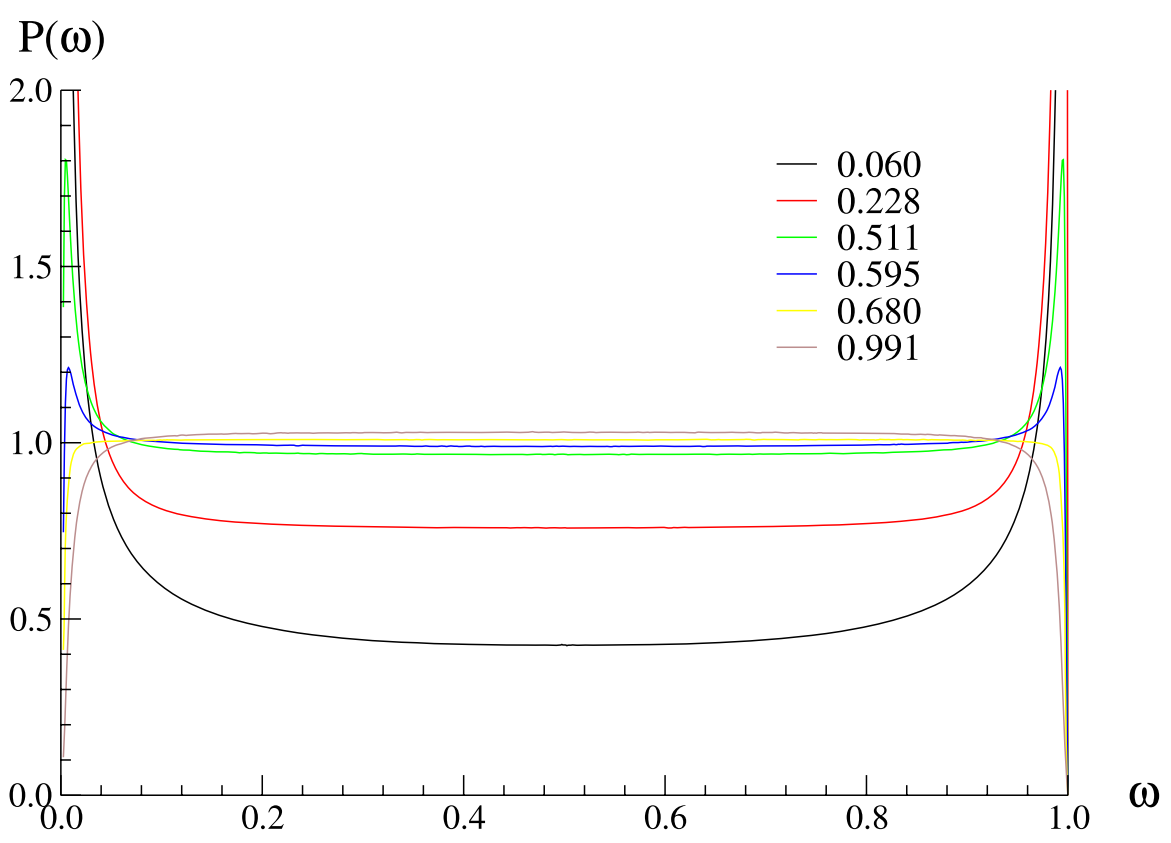

Figure 13. Two BMs in a sphere with a reflecting boundary. The distribution $P(\omega)$ in equation (72) for $L=100, r=5$ and different values of $x_{0} / L$.

where $B_{m}(\cdot)$ are the Bernoulli polynomials. The distribution in equation (74) is depicted in figure 14 for fixed $L$ and $D$, and different values of the ratio $x_{0} / L$. Note that here the situation is inverse to the one in which the target is situated in the origin-the most pronounced intermediate time power-law behavior is observed for $x_{0} / L \sim 1$ and is absent for small values of $x_{0} / L$. The normalized distribution $P(\omega)$ in this case has the form

$$
\begin{aligned}
P(\omega)=\frac{4 L^{2}}{\pi^{2} x_{0}^{2}} & \sum_{n, m=1}^{\infty}(-1)^{n+m} \frac{n m \sin \left(\pi n x_{0} / L\right) \sin \left(\pi m x_{0} / L\right)}{\left(\omega n^{2}+(1-\omega) m^{2}\right)^{2}} \\
& =-\left.\frac{\mathrm{i} L^{2}}{2 \pi x_{0}^{2}} \frac{1}{\omega^{3 / 2}(1-\omega)^{1 / 2}} \frac{\mathrm{d}}{\mathrm{d} y} \ln \left(\frac{\theta_{3}\left(x_{0}(1+\mathrm{i} y) / 2 L, \mathrm{e}^{-\pi y}\right)}{\theta_{3}\left(x_{0}(1-\mathrm{i} y) / 2 L, \mathrm{e}^{-\pi y}\right)}\right)\right|_{y=\sqrt{1-\omega / \omega}}
\end{aligned}
$$

where $\theta_{3}$ is the Jacobi theta function:

$$
\theta_{3}(v, q)=\sum_{m=-\infty}^{\infty} q^{n^{2}} \exp (2 \pi \mathrm{i} m v)
$$

The distribution in equation (76) is depicted in figure 15 for fixed $L$ and different values of $x_{0} / L$. We observe here a transition from a bell-shaped form with a maximum at $\omega=1 / 2$ and an M-shaped form with a minimum at $\omega=1 / 2$ and maxima close to 0 and 1 . The transition takes place at $x_{0} / L=0.643 \cdots$. Averaging equation (76) over the starting point $x_{0}$, we again find that $P_{\mathrm{av}}(\omega) \equiv$ 1. 
$\log \Psi(\tau)$

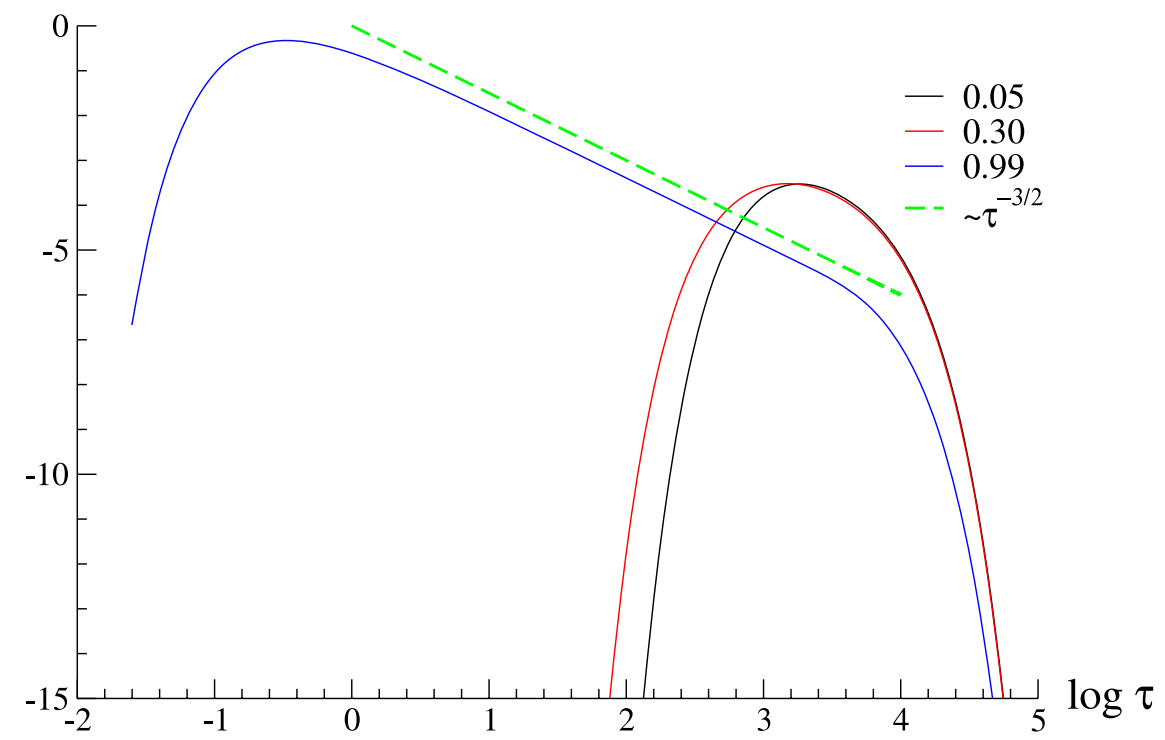

Figure 14. A BM starting at a distance $x_{0}$ from the origin of a sphere with an adsorbing boundary. The first passage time distribution $\Psi(\tau)$ in equation (74) for $L=100, D=1 / 2$ and different values of $x_{0} / L$.

\section{The distribution $P(\omega)$ for exponentially truncated first passage time distributions. Three and more non-communicating searchers}

We turn finally to the situation with more than two searchers whose first passage time distribution obeys an exponentially truncated form in equation (6). For $N=3$ we find the following general result:

$$
\begin{aligned}
P(\omega)=\frac{(b / 4 a)^{3 \mu / 2}}{2 K_{\mu}^{3}(2 \sqrt{a / b})} \frac{\omega^{\mu-1}}{(1-\omega)^{\mu+1}} \int_{0}^{\infty} x^{\mu} \mathrm{d} x J_{\mu-1}(x) \\
\times\left(x^{2}+\frac{4 a}{b \omega}\right)^{\mu} K_{\mu}^{2}\left(\sqrt{\frac{\omega}{1-\omega}\left(x^{2}+\frac{4 a}{b \omega}\right)}\right) .
\end{aligned}
$$

A straightforward analysis shows that $P(\omega)$ in equation (78) is always a bell-shaped function for $\mu \geq 1$. The most probable value $\omega_{m}$ is, however, always substantially less than $1 / 3$, approaching this value only when $\mu \rightarrow \infty$ or $b \rightarrow 0$.

The case $0<\mu<1$ is different. Focusing on $\mu=1 / 2$ (e.g. biased BMs on a semi-infinite line), for which equation (78) simplifies:

$$
P(\omega)=\frac{4}{\pi} \sqrt{\frac{a}{b}} \frac{\mathrm{e}^{6 \sqrt{a / b}}}{\omega(1-\omega) \sqrt{1+3 \omega}} K_{1}\left(2 \sqrt{\frac{a}{b} \frac{1+3 \omega}{\omega(1-\omega)}}\right),
$$

we discuss a sequence of different regimes which may be observed when $b / a$ is gradually varied, see figure 16 . For $b / a \ll 1, P(\omega)$ is peaked at $\omega_{m} \approx 1 / 3$. For larger $b / a, \omega_{m}$ moves towards the origin and $P\left(\omega_{m}\right)$ decreases (see the inset of figure 17). For yet larger $b / a, \omega_{m}$ keeps moving toward the origin but now $P\left(\omega_{m}\right)$ passes through a minimum and then starts 


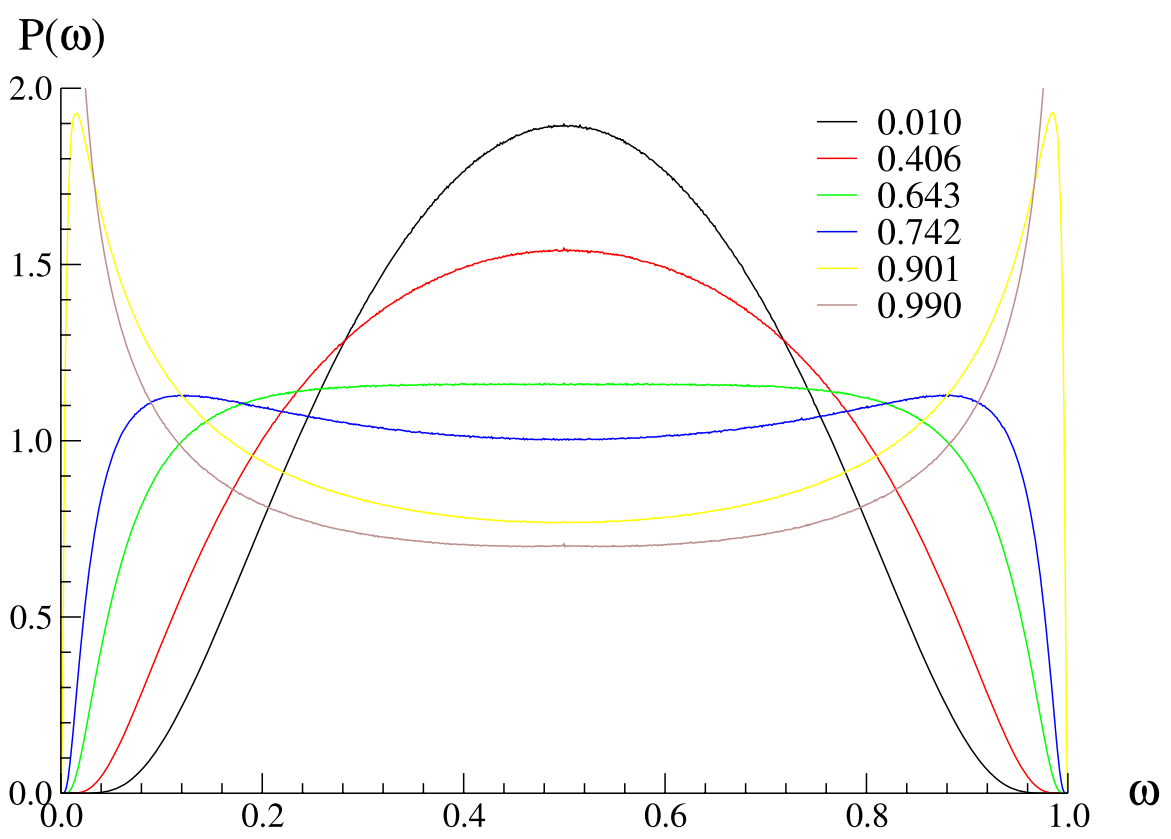

Figure 15. Two BMs starting at a distance $x_{0}$ from the origin of a sphere with an adsorbing boundary. The distribution $P(\omega)$ in equation (76) for $L=100$ and different values of $x_{0} / L$.

$P(\omega)$

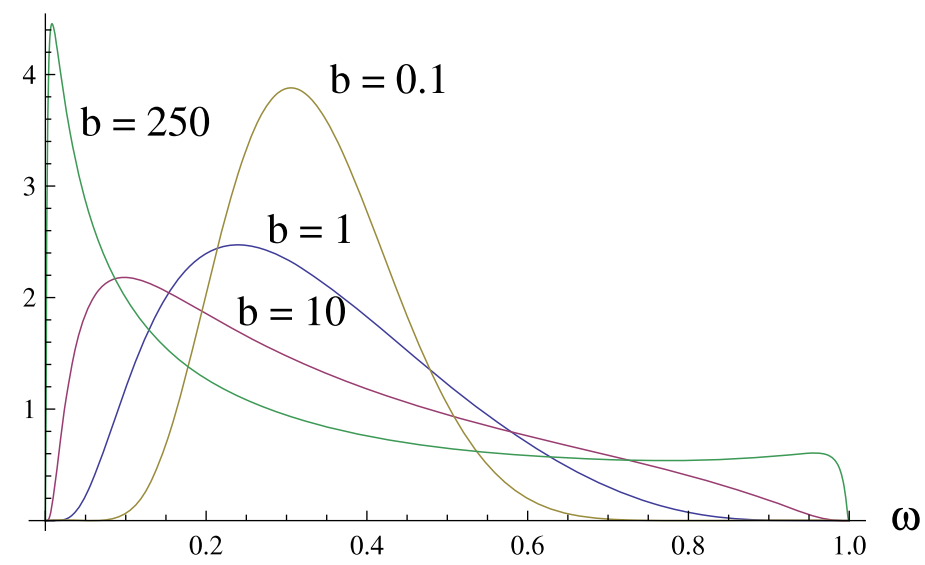

Figure 16. Three non-communicating Brownian searchers. $P(\omega)$ in equation (79) for different values of the parameter $b$ ( $a$ is set equal to 1$)$.

to grow. At $b / a \approx 140$ a second extremum emerges at $\omega \approx 0.84$ which then splits into a minimum and a maximum (see figure 17) so that $P(\omega)$ becomes bimodal. For still larger $b / a$, the minimum moves toward $\omega=1 / 2$, while the second maximum moves to $\omega=1$.

In fact, the result in equation (79) can be straightforwardly generalized for arbitrary $N$, yielding

$$
P(\omega)=\frac{2(N-1)}{\pi} \sqrt{\frac{a}{b}} \frac{\mathrm{e}^{2 N \sqrt{a / b}}}{\omega(1-\omega) \sqrt{(N-1)^{2} w+1-w}} K_{1}\left(2 \sqrt{\frac{a}{b}\left(\frac{1}{w}+\frac{(N-1)^{2}}{1-w}\right)}\right) .
$$


First passages for a search by a swarm of independent random searchers

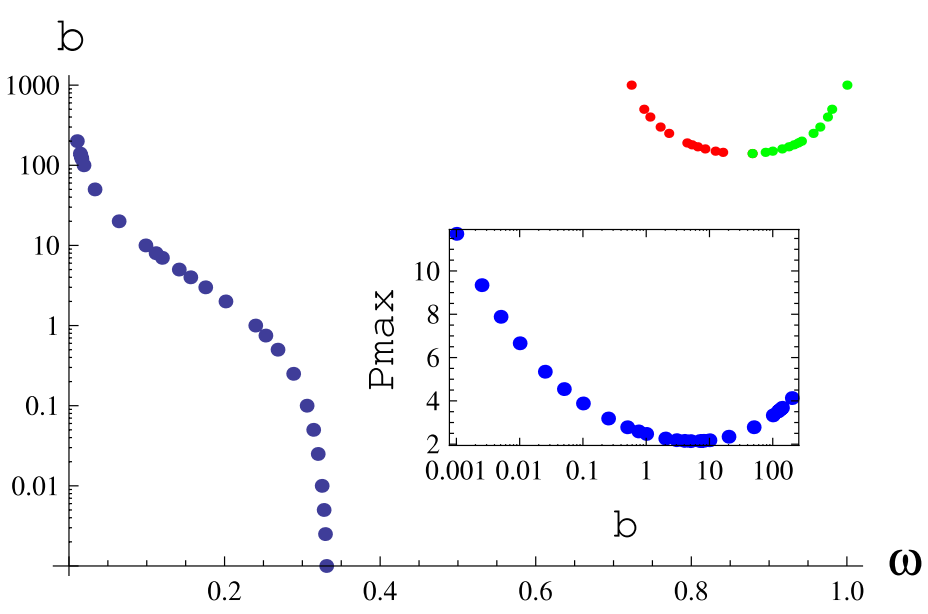

Figure 17. Three non-communicating Brownian searchers. The loci of the extrema of $P(\omega)$ in equation (79). Blue (green) circles define the position of the first (second) maximum. Red circles define the position of the minimum of $P(\omega)$. The inset shows the maximal value $P\left(\omega_{m}\right)$ versus $b$ ( $a$ is set equal to 1 ).

The distribution function in equation (80) shows essentially the same behavior as the one in equation (79); the only difference is that the critical values of the parameter $b / a$ at which $P_{\max }$ attains a minimal value or when the second maximum emerges depend on the number of searchers $N$.

\section{Conclusions}

To conclude, in this paper we have studied the distribution $P(\omega)$ of the random variable $\omega \sim \tau_{1} / \bar{\tau}, \bar{\tau}=N^{-1} \sum_{k=1}^{N} \tau_{k}$, where $\tau_{k}$ s are the first passage times to an immobile target by $N$ independent searchers, which start their random motion simultaneously from the same point in space. Hence, $\tau_{k} \mathrm{~s}$ are independent, identically distributed (with distribution $\Psi(\tau)$ ) random variables. Since $\omega$ equals, by definition, the realization-dependent first passage time of a given searcher relative to the realization-dependent ensemble-average first passage time of $N$ searchers, the distribution $P(\omega)$ can be viewed as a measure of the robustness of a given search algorithm and of the underlying random motion (space exploration), which also probes the validity of the mean first passage time of a single searcher as a proper and/or informative measure of the search efficiency.

We have considered two general forms of $\Psi(\tau)$ : the one in equation (4), which is appropriate for a search in unbounded domains and is characterized by a power-law longtime tail $\sim \tau^{-1-\mu}$, where the exponent $\mu$ encodes the specific details of the searchers' random motion, and an exponentially tempered form in equation (6), which is a plausible approximation for random motion in finite domains or a search assisted by a constant bias ('smell') toward the target.

We have shown that, for a non-truncated distribution $\Psi(\tau)$ in equation (4) with $\mu<1$, the distribution of the random variable $\omega$ has a characteristic U-shaped form so that the most probable values of $\omega$ are 0 and 1 . For $N=2$ the distribution is symmetric around $\omega=1 / 2$, with $\omega=1 / 2$ being the minimum of the distribution. This signifies that the 'symmetry' between two identical searchers is broken. For $N>2$ the distribution $P(\omega)$ is 
skewed (by a factor $N^{2}$ ) towards the small values of $\omega$ and can be expressed, in an explicit form, as a one-sided $\alpha$-stable distribution with $\alpha=2 \mu$. For $\mu \geq 1$, the distribution $P(\omega)$ has a bell-shaped form but the most probable value of $\omega$ is always less than the mean value $\langle\omega\rangle=1 / N$. The most probable and the mean values of $\omega$ coincide only when $\mu \rightarrow \infty$.

For the exponentially truncated $\Psi(\tau)$ in equation (6) the distribution $P(\omega)$ always has a bell-shaped form for $\mu \geq 1$. For $\mu<1$, however, the situation is more complicated and interesting. We realized first that for $N=2$ there exists some critical value of the parameter $y_{\mathrm{c}}(\mu)=b / a$, so that for $b / a<y_{\mathrm{c}}(\mu)$ the distribution $P(\omega)$ has a bell-shaped form, but for $b / a>y_{\mathrm{c}}(\mu)$ it attains an M-shaped form with $\omega=1 / 2$ being the least probable value and two maxima close to the edges of the interval. This signifies that, despite the fact that $\Psi(\tau)$ has moments of arbitrary order, two identical searchers will arrive for the first time at the target at distinctly different times. Consequently, in such a situation the mean first passage is not a proper measure of the search efficiency. We remember that such a form of $\Psi(\tau)$ is exact for a BM taking place on a semi-infinite one-dimensional line with a constant bias directed towards the target. For this physical situation, our result implies that two identical BMs, starting at some point $x_{0}$ and having the same drift velocity $v<0$ and the same diffusion coefficient $D$, will most likely arrive together at the target (the origin) if the Peclet number $P e=x_{0}|v| / 2 D$ exceeds some critical value $P e_{\mathrm{c}} \approx 0.666$. In contrast, if $P e<P e_{\mathrm{c}}$, an event that these two BMs arrive simultaneously at the target location is the least probable event.

Turning next to an unbiased $\mathrm{BM}$ on a finite interval $[0, L]$ we recall that $b$ should be proportional to $L^{2} / D$ and $a \sim x_{0}^{2} / D$. Hence, $\sqrt{a / b} \sim x_{0} / L$. This suggests a somewhat strange result that, for two unbiased identical BMs on a finite 1D interval, the modality of the distribution should depend on how far the starting point is from the reflecting boundary. Since $\Psi(\tau)$ in equation (6), which we have used for the derivation of this result, is an approximate form of the first passage time distribution, we have revisited this problem using exact forms of $\Psi(\tau)$ for a BM in 1D, 2D and 3D spherical domains with a reflecting boundary. We have shown that, indeed, the very shape (modality) of the distribution $P(\omega)$ depends on the ratio $x_{0} / L$.

We have realized that, for $x_{0} / L<\chi_{\mathrm{c}}(d)$, where $\chi_{\mathrm{c}}(d) \approx 0.61,0.64$ and 0.68 for $1 \mathrm{D}$, $2 \mathrm{D}$ and $3 \mathrm{D}$, respectively, $P(\omega)$ is an M-shaped function of $\omega$ with a minimum at $\omega=1 / 2$ so that here two unbiased identical BMs will most probably arrive at the target location for the first time at distinctly different times. For $x_{0} / L>\chi_{\mathrm{c}}(d)$, the distribution has a maximum at $\omega=1 / 2$ so that, mathematically, the most probable event is that two BMs arrive for the first time at the location of the target simultaneously. Note, however, that $P(\omega)$ is a 'bell-shaped' function only in 1D (although it is still rather broad) but in $2 \mathrm{D}$ and $3 \mathrm{D}, P(\omega)$ is nearly flat in an extended region around the maximum and rather abruptly vanishes in the vicinity of the edges of the interval. This signifies that here sample-to-sample fluctuations are very significant. In the case when the starting point $x_{0}$ of two BMs is uniformly distributed within the domain (outside the target), we found that $P(\omega) \equiv 1$. This allows us to conclude that in neither of these well-studied situations (apart from, with some reservations, the $1 \mathrm{D}$ case with $x_{0} / L \sim 1$ ) the mean first passage time of an individual searcher can be considered as a robust measure of the search process efficiency.

We argue that a similar behavior will take place in finite $1 \mathrm{D}$ systems for fractional BM with arbitrary Hurst index $H$ or for $\alpha$-stable Lévy flights with $0<\alpha<1$ and, 


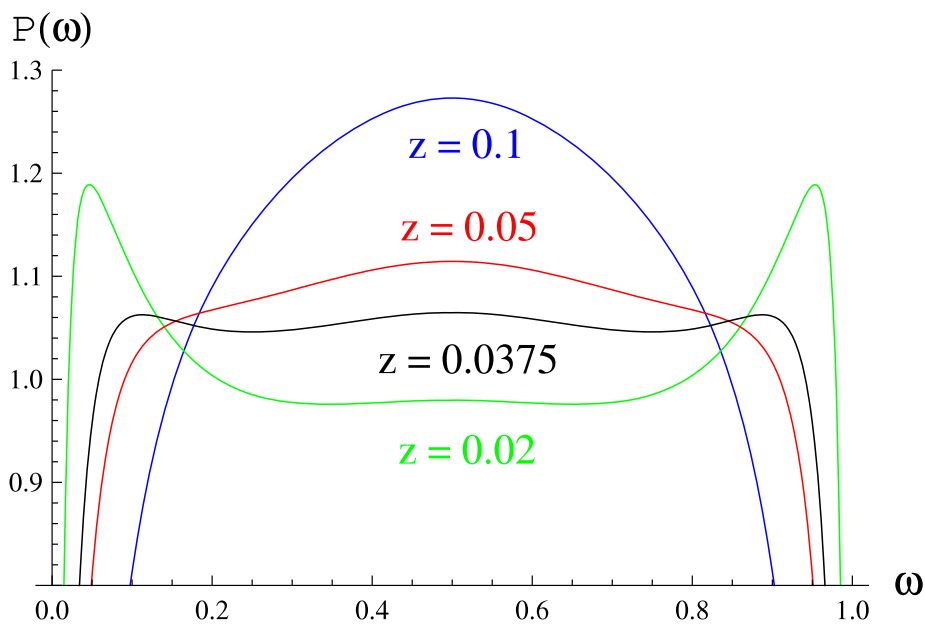

Figure 18. $P(\omega)$ in equation (82) for a faster-than-exponential truncation of the intermediate power-law behavior. Here the parameter $z=2 a / b$.

more generally, for finite systems of (not necessarily integer) dimension $d_{\mathrm{f}}$ with fractal dimension $d_{\mathrm{w}}$ of random motion trajectories given that (a) $d_{\mathrm{f}} \leq d_{\mathrm{w}}$ (compact exploration, $\left.\mu=1-d_{\mathrm{f}} / d_{\mathrm{w}}<1\right)$ or (b) $d_{\mathrm{f}}>d_{\mathrm{w}}$ (non-compact exploration, $\mu=d_{\mathrm{f}} / d_{\mathrm{w}}-1$ ) but $d_{\mathrm{f}}<2 d_{\mathrm{w}}$.

We have evaluated $P(\omega)$ in the case of three and more searchers with the exponentially truncated first passage time distribution in equation (6). We have shown that for $\mu>1$ the distribution $P(\omega)$ is a bell-shaped function of $\omega$ for any value of $b / a$. Next, we have demonstrated that for very small values of the ratio $b / a$ the distribution $P(\omega)$ has a bellshaped form with a maximum close to $1 / N$. Further on, we have predicted the following sequence of regimes which can be observed upon gradually increasing $b / a$ (the starting point $x_{0}$ of $N$ searchers is moving towards the location of the target): the most probable value $\omega_{m}$ of $\omega$ moves toward the origin. The value of the maximum, $P\left(\omega_{m}\right)$, first decreases, passes through a minimal value and then starts to increase. At a certain threshold value of $b / a$, a second extremum emerges in the vicinity of $\omega=1$, which then splits into a minimum and a maximum so that the distribution $P(\omega)$ becomes a skewed M-shaped one. This signifies, as well, that for such a situation the mean first passage time of a given searcher is not a representative characteristic of the search process.

As a final observation, we note that one may encounter a power-law distribution with a more abrupt truncation, compared to the exponential function in equation (6), say, a bounded power-law (see [31]) or a power-law tempered from both sides by a Gaussian function:

$$
\Psi(\tau)=\frac{(a b)^{\mu / 2}}{K_{\mu / 2}(2 a / b)} \exp \left(-\frac{a^{2}}{\tau^{2}}\right) \frac{1}{\tau^{1+\mu}} \exp \left(-\frac{\tau^{2}}{b^{2}}\right) .
$$

In this case, the distribution of the random variable $\omega$ is given by

$$
P(\omega)=\frac{1}{K_{-\mu / 2}^{2}(2 a / b)} \frac{1}{\omega(1-\omega)} K_{-\mu}\left(2 \frac{a\left(\omega^{2}+(1-\omega)^{2}\right)}{b \omega(1-\omega)}\right) .
$$

In figure 18 we plot the result in equation (82) for $\mu=\frac{1}{2}$ and different values of the parameter $z=2 a / b$. One finds that $P(\omega)$ in equation $(82)$ is always a bell-shaped 
First passages for a search by a swarm of independent random searchers

function for $\mu \geq 1$. For $\mu<1$, depending on the value of $z=2 a / b$, it may have a unimodal or a three-modal form.

\section{Acknowledgments}

The authors wish to thank O Bénichou, C Godrèche, S N Majumdar, I M Sokolov and M Vergassola for helpful discussions.

\section{References}

[1] Stephens D W and Krebs J R, 1986 Foraging Theory (Princeton, NJ: Princeton University Press)

[2] Bell J W, 1991 Searching Behavior, The Behavioural Ecology of Finding Resources (Chapman and Hall Animal Behaviour Series) (London: Chapman and Hall)

[3] Klafter J, Shlesinger M and Zumofen G, 1996 Phys. Today 4933

[4] see, e.g., Loverdo C, Bénichou O, Moreau M and Voituriez R, 2008 Nature Phys. 4134 Bénichou O, Coppey M, Moreau M and Oshanin G, 2005 J. Chem. Phys. 123194506 and references therein

[5] Ward M J and Keller J B, 1993 SIAM J. Appl. Math. 53770 Grigoriev I V, Makhnovskii Y A, Bereshkovskii A M and Zitserman V Y, 2002 J. Chem. Phys. 1169574 Bénichou O and Voituriez R, 2008 Phys. Rev. Lett. 100168105 Bénichou O et al, 2010 Phys. Rev. Lett. 105150606

[6] Oshanin G, Tamm M and Vasilyev O, 2010 J. Chem. Phys. 132235101

[7] Berg O G, Winter R B and Von Hippel P H, 1981 Biochemistry 206929

Coppey M, Bénichou O, Voituriez R and Moreau M, 2004 Biophys. J. 871640

Slutsky M and Mirny L, 2004 Biophys. J. 874021

Sokolov I M, Metzler R, Pant K and Williams M C, 2005 Biophys. J 89895

$\mathrm{Hu}$ L, Grosberg A Yu and Bruinsma R, 2008 Biophys. J. 951151

van den Broek B et al, 2008 Proc. Nat. Acad. Sci. 10515738

Bonnet I et al, 2008 Nucleic Acids Res. 364118

Lomholt M A et al, 2009 Proc. Nat. Acad. Sci. 1068204

[8] Kabatiansky G and Oshanin G, 2009 J. Phys. A: Math. Theor. 42434016

[9] Oshanin G, Vasilyev O, Krapivsky P and Klafter J, 2009 Proc. Nat. Acad. Sci. 10613696

[10] Kamimura A and Ohira T, 2010 New J. Phys. 12053013 Vicsek T, 2010 Nature 46643

[11] Chun M M and Wolfe J M, 1996 Cognit. Physiol. 3039 Horowitz T S and Wolfe J M, 1998 Nature 394575

[12] Bénichou O et al, 2000 Phys. Rev. Lett. 84511 Bénichou O et al, 2001 Phys. Rev. B 63235413 Weber S C, Spakowitz A J and Theriot J A, 2010 Phys. Rev. Lett. 104238102 Jeon J-H et al, 2011 Phys. Rev. Lett. 106048103 Oshanin G and Mejía-Monasterio C, 2011 Soft Matter 7993

[13] Wadhams G H and Armitage J P, 2004 Nat. Rev. Mol. Cell Biol. 51024 de Gennes P G, 2004 Eur. Biophys. J. 33691 Vergassola M, Villermaux E and Shraiman B I, 2007 Nature 445406 Celani A and Vergassola M, 2010 Proc. Nat. Acad. Sci. 1071391 Revelli J A, Rojo F, Budde C E and Wio H S, 2010 J. Phys. A: Math. Theor. 43195001 Sengupta A, Kruppa T and Löwen H, 2011 Phys. Rev. E 83031914

[14] Masson J B, Bailly-Bechet M and Vergassola M, 2009 J. Phys. A: Math. Theor. 42434009

[15] Gelenbe E, 2010 Phys. Rev. E 82061112

[16] Evans M R and Majumdar S N, 2011 Phys. Rev. Lett. 106160601

[17] Stone L D, 1989 Theory of Optimal Search (Arlington, VA: Operations Research of America)

[18] Edwards A M et al, 2007 Nature 4491044

[19] Ramos-Fernandez G et al, 2003 Behav. Ecol. Sociobiol. 55223 Boyer D et al, 2006 Proc. Biol. Sci. 2731743

[20] Kramer D L and MacLaughlin R L, 2001 Am. Zool. 41137

[21] Bénichou O et al, 2005 Phys. Rev. Lett. 94198101

Bénichou O, Coppey M, Moreau M and Voituriez R, 2006 Europhys. Lett. 75349 Moreau M, Bénichou O, Loverdo C and Voituriez R, 2009 J. Stat. Mech. P12006

[22] Oshanin G, Wio H S, Lindenberg K and Burlatsky S F, 2007 J. Phys.: Condens. Matter 19065142 
Oshanin G, Lindenberg K, Wio H S and Burlatsky S, 2009 J. Phys. A: Math. Theor. 42434008 Rojo F et al, 2010 J. Phys. A: Math. Theor. 43345001

Rojo F, Pury P A and Budde C E, 2011 Phys. Rev. E 83011116

[23] Newby J and Bressloff P C, 2010 J. Stat. Mech. P04014

[24] Portillo I G, Campos D and Méndez V, 2011 J. Stat. Mech. P02033

[25] Lomholt M A, Koren T, Metzler R and Klafter J, 2008 Proc. Nat. Acad. Sci. 10511055

[26] Eliazar I, 2005 Physica A 356207

[27] Oshanin G and Redner S, 2009 Europhys. Lett. 8510008

[28] Eliazar I and Sokolov I M, 2010 J. Phys. A: Math. Theor. 43055001

[29] Sokolov I M and Eliazar I I, 2010 Phys. Rev. E 81026107

[30] Oshanin G and Schehr G, Quantitative Finance, 2010 arXiv:1005.1760v1 at press

[31] Oshanin G, Holovatch Y and Schehr G, 2011 Physica A at press, arXiv:1106.4710v1

[32] Majumdar S N, 1999 Curr. Sci. 77370

[33] Redner S, 2001 A Guide to First-Passage Processes (Cambridge: Cambridge University Press)

[34] Sparre Andersen E, 1953 Math. Scand. 1263 Sparre Andersen E, 1954 Math. Scand. 2195

[35] Chechkin A et al, 2003 J. Phys. A: Math. Gen. 36 L537 Koren T et al, 2007 Phys. Rev. Lett. 99160602

[36] Hansen A, Engoy T and Maloy K J, 1994 Fractals 2527 Maslov S, Paczuski M and Bak P, 1994 Phys. Rev. Lett. 732162 Ding M and Yang W, 1995 Phys. Rev. E 52207

Krug J et al, 1997 Phys. Rev. E 562702 Molchan G M, 1999 Commun. Math. Phys. 20597

[37] de Gennes P G, 1982 J. Chem. Phys. 763316

[38] Oshanin G S, Burlatsky S F and Ovchinnikov A A, 1989 Phys. Lett. A 139245

[39] Oshanin G, Moreau M and Burlatsky S F, 1994 Adv. Colloid Interface Sci. 491

[40] Gershtein G L and Mandelbrot B B, 1964 Biophys. J. 441

[41] Lima A R, Moukarzel C F, Grosse I and Penna T J P, 2000 Phys. Rev. E 612267

[42] Flyvbjerg H, Sneppen K and Bak P, 1993 Phys. Rev. Lett. 714087

[43] Oshanin G, Mogutov A and Moreau M, 1993 J. Stat. Phys. 73379 Oshanin G, Burlatsky S F, Moreau M and Gaveau B, 1993 Chem. Phys. 177803 Monthus C and Comtet A, 1994 J. Physique I 4635

[44] Lindenberg K, Seshadri V, Shuler K E and Weiss G H, 1980 J. Stat. Phys. 2311

[45] Krapivsky P L, Majumdar S N and Rosso A, 2010 J. Phys. A: Math. Theor. 43315001 Redner S and Krapivsky P L, 1999 Am. J. Phys. 671277

[46] Bénichou O et al, 2003 J. Phys. A: Math. Gen. 367225

[47] Yuste S B, Acedo L and Lindenberg K, 2001 Phys. Rev. E 64052102

[48] Bénichou O, Kafri Y, Sheinman M and Voituriez R, 2009 Phys. Rev. Lett. 103138102

[49] Majumdar S N and Comtet A, 2002 Phys. Rev. Lett. 89060601 Sabhapandit S, Majumdar S N and Comtet A, 2006 Phys. Rev. E 73051102

[50] Hughes B D, 1995 Random Walks and Random Environments (Oxford: Clarendon)

[51] Kingman J F C, 1963 Acta Math. 10911

[52] Kolokoltsov V, 2000 Proc. Lond. Math. Soc. 80725

[53] Lévy P, 1939 Comput. Math. 7283

[54] Govorun E N et al, 2001 Phys. Rev. E 64 040903(R) 